The Annals of Probability

2002, Vol. 30, No. 2, 605-65

\title{
FLUCTUATIONS OF THE FREE ENERGY IN THE REM AND THE $p$-SPIN SK MODELS
}

\author{
By Anton Bovier, Irina Kurkova And MatThias Löwe \\ Weierstrass-Institut für Angewandte Analysis und Stochastik, \\ Université Paris 6 and Catholic University Nijmegen
}

\begin{abstract}
We consider the random fluctuations of the free energy in the $p$-spin version of the Sherrington-Kirkpatrick (SK) model in the high-temperature regime. Using the martingale approach of Comets and Neveu as used in the standard SK model combined with truncation techniques inspired by a recent paper by Talagrand on the $p$-spin version, we prove that the random corrections to the free energy are on a scale $N^{-(p-2) / 2}$ only and, after proper rescaling, converge to a standard Gaussian random variable. This is shown to hold for all values of the inverse temperature, $\beta$, smaller than a critical $\beta_{p}$. We also show that $\beta_{p} \rightarrow \sqrt{2 \ln 2}$ as $p \uparrow+\infty$. Additionally, we study the formal $p \uparrow+\infty$ limit of these models, the random energy model. Here we compute the precise limit theorem for the (properly rescaled) partition function at all temperatures. For $\beta<\sqrt{2 \ln 2}$, fluctuations are found at an exponentially small scale, with two distinct limit laws above and below a second critical value $\sqrt{\ln 2 / 2}$ : for $\beta$ up to that value the rescaled fluctuations are Gaussian, while below that there are non-Gaussian fluctuations driven by the Poisson process of the extreme values of the random energies. For $\beta$ larger than the critical $\sqrt{2 \ln 2}$, the fluctuations of the logarithm of the partition function are on a scale of 1 and are expressed in terms of the Poisson process of extremes. At the critical temperature, the partition function divided by its expectation converges to $1 / 2$.
\end{abstract}

1. Introduction. In recent years it has become increasingly clear that a problem of central importance for the understanding of disordered spin systems is the control of random fluctuations of thermodynamic quantities (see [2], [7], [19] and [27]). Unfortunately, precise control of such quantities is very hard to come by. Concentration of measure techniques [26] have been realized to be efficient tools to get upper bounds ([5] and [6]), but lower bounds or exact limit theorems are scarce. One of these examples is the Sherrington-Kirkpatrick (SK) model in the high-temperature phase, where a central limit theorem for the free energy was proven first by Aizenman, Lebowitz and Ruelle [1], using cluster expansion techniques, and later by Comets and Neveu [9], making use of martingale methods and stochastic calculus. Their methods have been extended to a few related cases later (see [3] and [28]). In the present paper we want to continue this effort by investigating a large class of natural generalization of the SK model, the so-called $p$-spin

Received July 2000; revised April 2001.

AMS 2000 subject classifications. 82C44, 60K 35.

Key words and phrases. Spin glasses, Sherrington-Kirkpatrick model, p-spin model, random energy model, central limit theorem, extreme values, martingales. 
SK models (see, e.g., [15] and [20]) and their $p \uparrow+\infty$ limit, the random energy model (REM).

For our present purposes it is natural to consider the class of models we study as Gaussian processes on the hypercube $\delta_{N}=\{-1,1\}^{N}$. We will always denote the corner of $s_{N}$ by $\sigma$; for historical reasons they are called spin configurations. A Gaussian process $X$ on $\delta_{N}$ is characterized completely by its mean and covariance function. The processes we consider will always be assumed to have mean 0 and covariances

$$
\mathbb{E} X_{\sigma} X_{\sigma^{\prime}} \equiv f_{N}\left(R_{N}\left(\sigma, \sigma^{\prime}\right)\right),
$$

where $R_{N}\left(\sigma, \sigma^{\prime}\right) \equiv N^{-1}\left(\sigma \cdot \sigma^{\prime}\right) \equiv N^{-1} \sum_{i=1}^{N} \sigma_{i} \sigma_{i}^{\prime}$ is the so-called overlap [this overlap is related to the Hamming distance $d_{\text {Ham }}$ by $d_{\text {Ham }}\left(\sigma, \sigma^{\prime}\right)=N(1-$ $\left.R_{N}\left(\sigma, \sigma^{\prime}\right)\right) / 2$ ]. In this paper we will investigate the case where

$$
f_{N}(x)=f_{N}^{p}(x):=\sum_{k=0}^{[p / 2]} \alpha_{p-2 k}(N) x^{p-2 k},
$$

where the coefficients satisfy $\alpha_{p}(N)=1$ and $\alpha_{p-2 k}(N)=O\left(N^{-k}\right)$. The reason for this complicated choice of covariance function is due to the fact that in the commonly used form of the $p$-spin interaction version of the SherringtonKirkpatrick model, the corresponding process is represented as

$$
X_{\sigma}=\left(\begin{array}{c}
N \\
p
\end{array}\right)^{-1 / 2} \sum_{i_{1}<i_{2}<\cdots<i_{p}} J_{i_{1}, i_{2}, \ldots, i_{p}} \sigma_{i_{1}} \sigma_{i_{2}} \cdots \sigma_{i_{p}},
$$

with $J_{i_{1}, \ldots, i_{p}}$ a family of $\left(\begin{array}{l}N \\ p\end{array}\right)$ i.i.d. standard Gaussian random variables. Computing the covariance of this process, one finds indeed an expression of the form (1.2). While we are mainly interested in this particular case, we will discuss the general case in Appendix A. Note that there is a certain ambiguity in the definition of the model with regard to whether or not coinciding indices in the sum in (1.3) are or are not excluded. For example, one may choose the simpler looking version

$$
\tilde{X}_{\sigma} \equiv N^{-p / 2} \sum_{1 \leq i_{1}, \ldots, i_{p} \leq N} J_{i_{1}, i_{2}, \ldots, i_{p}} \sigma_{i_{1}} \cdots \sigma_{i_{p}} .
$$

For this model the covariance is simply $\operatorname{cov}\left(\tilde{X}_{\sigma}, \tilde{X}_{\sigma^{\prime}}\right)=R_{N}\left(\sigma, \sigma^{\prime}\right)^{p}$. While the two models are identical on the level of the thermodynamic functions, we will see that the scales of fluctuations are quite different.

We also study the formal limit of $p$-spin SK models as $p \uparrow+\infty$, the random energy model (REM). In this model the random variables $X_{\sigma}$ are independent standard normal.

Given such a Gaussian process, our main object of interest is the so-called partition function

$$
Z_{\beta, N} \equiv \mathbb{E}_{\sigma} e^{\beta \sqrt{N} X_{\sigma}} \equiv 2^{-N} \sum_{\sigma \in S_{N}} e^{\beta \sqrt{N} X_{\sigma}}
$$


The quantities $e^{\beta \sqrt{N} X_{\sigma}}$ are called Boltzmann weights, the parameter $\beta \in \mathbb{R}_{+}$ is known as the inverse temperature and $H_{N}(\sigma) \equiv \sqrt{N} X_{\sigma}$ is (minus) the Hamiltonian in statistical mechanics. The $Z_{\beta, N}$ are random variables, and we are primarily interested in their behavior as $N \rightarrow \infty$. In statistical mechanics, it is customary to introduce the so-called free energy

$$
F_{\beta, N} \equiv-\frac{1}{\beta N} \ln Z_{\beta, N}
$$

It is easy to prove in all the models we consider here that, for all values of $\beta, F_{\beta, N}$ is a self-averaging quantity, that is,

$$
\lim _{N \uparrow+\infty}\left|F_{\beta, N}-\mathbb{E} F_{\beta, N}\right|=0 \text { a.s. }
$$

It is, however, not known in general whether the so-called quenched free energy $\mathbb{E} F_{\beta, N}$ converges to a limit as $N \rightarrow \infty$. This has, however, been proven for sufficiently small values of $\beta$. More precisely, we have the following result.

TheOrem 1.1. Define $\tilde{\beta}_{2}=1$ and, for $p>2$,

$$
\tilde{\beta}_{p}^{2} \equiv \inf _{0<m<1}\left(1+m^{-p}\right) \phi(m)
$$

where

$$
\phi(m) \equiv[(1-m) \ln (1-m)+(1+m) \ln (1+m)] / 2 .
$$

Then, for all $\beta<\tilde{\beta}_{p}$,

$$
\lim _{N \uparrow+\infty} \mathbb{E} F_{\beta, N, p}=-\beta / 2 .
$$

REMARK. For $p=2$ this result was first proven in [1]. A very simple proof was later given by Talagrand [26]. Comets [8] showed that the value $\tilde{\beta}_{2}=1$ is optimal in the sense that (1.9) fails for $\beta>1$. The result for $p \geq 3$ is due to Talagrand [27]. It is clear that in all cases (1.9) will fail for $\beta \geq \sqrt{2 \ln 2}$, which by a more elaborate computation can be improved to $\beta \geq \sqrt{2 \ln 2}\left(1-2^{-c_{p} p}\right)$ with $c_{p}<5$, for $p$ large [4]. On the other hand, a simple calculation shows that $\tilde{\beta}_{p} \sim$ $\sqrt{2 \ln 2}\left(1-2^{-p} / 2 \ln 2\right)$. One should note that to get (1.9) up to a value so close to $\sqrt{2 \ln 2}$ required a substantial modification of the original argument of [26], namely, the use of a "truncated" second-moment method. Such a truncation will also be the main difficulty in obtaining our results (for similar reasons, slightly different truncations were also used by Toubol [28] (and probably first) in the study of the CLT for the SK model with vector-valued spins).

In the case of the REM, it is well known that the critical inverse temperature $\tilde{\beta}_{\text {REM }}=\sqrt{2 \ln 2}$ and that $[11]$

$$
\lim _{N \uparrow+\infty} \mathbb{E} F_{\beta, N, \operatorname{REM}}= \begin{cases}-\beta / 2, & \text { if } \beta \leq \sqrt{2 \ln 2}, \\ -\sqrt{2 \ln 2}+\beta^{-1} \ln 2, & \text { if } \beta \geq \sqrt{2 \ln 2} .\end{cases}
$$


As observed already in [10] (a rigorous proof follows easily from the results contained in [27] (Talagrand, private communication)), one has that

(1.11) $\lim _{p \uparrow+\infty} \limsup _{N \uparrow+\infty} \mathbb{E} F_{\beta, N, p}=\lim _{p \uparrow+\infty} \liminf _{N \uparrow+\infty} \mathbb{E} F_{\beta, N, p}=\lim _{N \uparrow+\infty} \mathbb{E} F_{\beta, N, \text { REM }}$

In this paper we will control the fluctuations of the free energy in (essentially) all of the domain of parameters $\beta$, $p$, where the limit is known to exist, that is, the high-temperature regions of the $p$-spin models and the entire temperature range in the REM. Although the REM is rather singular and the techniques used for that case are totally different from those we will use for the $p$-spin models, we felt it would be instructive to include this singular limiting case in this paper. Moreover, it turns out that in spite of the heavy investigation the REM has enjoyed over the years (see [10-14], [21] and [23]), no precise fluctuation results for the free energy are available in the literature (see the remark following Theorem 1.6). Finally, we are convinced that the reader will be rather surprised by the rich structure the fluctuation behavior this model exhibits.

Let us now state our results. We begin with the $p$-spin SK models.

THEOREM 1.2. Consider the $p$-spin SK models defined by (1.3), $p \geq 3$. There exists $\beta_{p}>0$ such that, for all $\beta<\beta_{p}$,

$$
N^{(p-2) / 2} \ln \frac{Z_{\beta, N}}{\mathbb{E} Z_{\beta, N}} \stackrel{D}{\rightarrow} \mathcal{N}\left(0, \frac{\beta^{4} p !}{2}\right) .
$$

REMARK. Note that the result of [1] and [8] in the case $p=2$ is not obtained by setting $p=2$ in (1.12). In particular, in that case the limit has a finite mean. The reader might wonder why in the case $p>2$ the limit has mean 0 even though we do not explicitly center our process. But this is actually easy to understand: $Z_{\beta, N} / \mathbb{E} Z_{\beta, N}$ has mean 1 and variance $\sim N^{-(p-2)}$. Now

$$
\begin{aligned}
\ln \left(Z_{\beta, N} / \mathbb{E} Z_{\beta, N}\right) & =\ln \left(1+\left(Z_{\beta, N} / \mathbb{E} Z_{\beta, N}-1\right)\right) \\
& =\left(Z_{\beta, N} / \mathbb{E} Z_{\beta, N}-1\right)+O\left(\left(Z_{\beta, N} / \mathbb{E} Z_{\beta, N}-1\right)^{2}\right),
\end{aligned}
$$

and so the mean of the left-hand side of (1.12), $N^{(p-2) / 2} \mathbb{E} \ln \left(Z_{\beta, N} / \mathbb{E} Z_{\beta, N}\right)=$ $O\left(N^{-(p-2) / 2}\right)$, tends to 0 .

The value of $\beta_{p}$ can be estimated reasonably well. To state a lower bound on $\beta_{p}$, however, we need some notation. We define the functions

$$
\begin{aligned}
I\left(m_{1}, m_{2}, m_{3}\right)=\frac{1}{4} & \left(\left(1+m_{1}+m_{2}+m_{3}\right) \ln \left(1+m_{1}+m_{2}+m_{3}\right)\right. \\
& +\left(1-m_{1}-m_{2}+m_{3}\right) \ln \left(1-m_{1}-m_{2}+m_{3}\right) \\
& +\left(1+m_{1}-m_{2}-m_{3}\right) \ln \left(1+m_{1}-m_{2}-m_{3}\right) \\
& \left.+\left(1-m_{1}+m_{2}-m_{3}\right) \ln \left(1-m_{1}+m_{2}-m_{3}\right)\right),
\end{aligned}
$$




$$
\begin{aligned}
S\left(m_{1}, m_{2}, m_{3}\right)= & {\left[\left(1+\frac{m_{1}-m_{2} m_{3}}{1-m_{3}^{2}}\right)^{2}+\left(1+\frac{m_{2}-m_{1} m_{3}}{1-m_{3}^{2}}\right)^{2}\right.} \\
& \left.+2 m_{3}\left(1+\frac{m_{1}-m_{2} m_{3}}{1-m_{3}^{2}}\right)\left(1+\frac{m_{2}-m_{1} m_{3}}{1-m_{3}^{2}}\right)\right]^{1 / 2},
\end{aligned}
$$

$$
R\left(m_{1}, m_{2}, m_{3}\right)=\frac{2 m_{1} m_{2} m_{3}-m_{1}^{2}-m_{2}^{2}}{2\left(1-m_{3}^{2}\right)}
$$

and

$$
\begin{aligned}
& U_{p}\left(m_{1}, m_{2}, m_{3}\right) \\
& \begin{aligned}
=I\left(m_{1}, m_{2}, m_{3}\right)\left(1+m_{3}^{p}\right)[ & S\left(m_{1}^{p}, m_{2}^{p}, m_{3}^{p}\right) \sqrt{2+2 m_{3}^{p}} \\
& \left.+R\left(m_{1}^{p}, m_{2}^{p}, m_{3}^{p}\right)\left(1+m_{3}^{p}\right)-\left(2+m_{3}^{p}\right)\right]^{-1}
\end{aligned}
\end{aligned}
$$

on the set

$$
\begin{aligned}
\mathcal{A} \equiv\left\{m_{1}, m_{2}, m_{3} \in[-1,1]^{3} \mid 1-m_{1}-m_{2}+m_{3}>0,\right. \\
\left.1-m_{1}+m_{2}-m_{3}>0,1+m_{1}-m_{2}-m_{3}>0\right\} .
\end{aligned}
$$

Note that the function $I\left(m_{1}, m_{2}, m_{3}\right)$ is symmetric in $m_{1}, m_{2}$ and $m_{3}$ and that $S\left(m_{1}, m_{2}, m_{3}\right), R\left(m_{1}, m_{2}, m_{3}\right)$ and $U_{p}\left(m_{1}, m_{2}, m_{3}\right)$ are symmetric in $m_{1}$ and $m_{2}$. Let

$$
\begin{aligned}
& Y_{p}\left(m_{1}, m_{2}, m_{3}\right)=\max \left\{I\left(m_{1}, m_{2}, m_{3}\right)\left(\frac{2}{3}+\frac{1}{m_{1}^{p}+m_{2}^{p}+m_{3}^{p}}\right),\right. \\
& \left.U_{p}\left(m_{1}, m_{2}, m_{3}\right), U_{p}\left(m_{1}, m_{3}, m_{2}\right), U_{p}\left(m_{2}, m_{3}, m_{1}\right)\right\} .
\end{aligned}
$$

With this notation we have:

THEOREM 1.3. Let $p>2$. Then

$$
\inf _{m_{1}, m_{2}, m_{3} \in \mathcal{A}} Y_{p}\left(m_{1}, m_{2}, m_{3}\right) \leq \beta_{p}^{2}<2 \ln 2 .
$$

In particular,

$$
\lim _{p \uparrow+\infty} \beta_{p}^{2}=2 \ln 2 .
$$

REMARK. Observe that the variational problem that gives the lower bound on $\beta_{p}$ [i.e., (1.19)] is different from that one determining the lower bound on the critical temperature in Theorem 1.1. This is due to the fact that the former requires estimates on third moments, while second-moment methods suffice for the latter. One would, of course, expect that the true values should be the same in both cases. The point is, however, that both bounds converge to the correct value $\sqrt{2 \ln 2}$ in the limit $p \uparrow \infty$. 
We would also like to point out that the scaling factor $N^{(p-2) / 2}$ in Theorem 1.2 is on the order of $\left(\mathbb{E}_{\sigma, \sigma^{\prime}} \operatorname{cov}\left(\sqrt{N} X_{\sigma}, \sqrt{N} X_{\sigma^{\prime}}\right)^{n}\right)^{-1 / 2}\left(\right.$ where $\mathbb{E}_{\sigma, \sigma^{\prime}} \equiv \mathbb{E}_{\sigma} \mathbb{E}_{\sigma^{\prime}}$ stands for the expectation with respect to the product measure of two independent copies $\sigma$ and $\sigma^{\prime}$ of the spin variables), where $n \geq 1$ is the minimal value for which the condition $\mathbb{E}_{\sigma, \sigma^{\prime}} \operatorname{cov}\left(\sqrt{N} X_{\sigma}, \sqrt{N} X_{\sigma^{\prime}}\right)^{n} \neq 0$ holds. For the Gaussian process (1.3), $n=2$, since

$$
\begin{aligned}
& \mathbb{E}_{\sigma, \sigma^{\prime}} \operatorname{cov}\left(\sqrt{N} X_{\sigma}, \sqrt{N} X_{\sigma^{\prime}}\right) \\
& \quad=N\left(\begin{array}{c}
N \\
p
\end{array}\right)^{-1} \sum_{i_{1}<i_{2}<\cdots<i_{p}} \mathbb{E}_{\sigma, \sigma^{\prime}} \sigma_{i_{1}} \sigma_{i_{1}}^{\prime} \cdots \sigma_{i_{p}} \sigma_{i_{p}}^{\prime}=0,
\end{aligned}
$$

while

$$
\mathbb{E}_{\sigma, \sigma^{\prime}}\left(\operatorname{cov}\left(\sqrt{N} X_{\sigma}, \sqrt{N} X_{\sigma^{\prime}}\right)\right)^{2}=N^{2}\left(\begin{array}{c}
N \\
p
\end{array}\right)^{-2} \sum_{i_{1}<i_{2}<\cdots<i_{p}} 1=N^{2}\left(\begin{array}{c}
N \\
p
\end{array}\right)^{-1}
$$

Note that, for the model defined by (1.4),

$$
\begin{aligned}
\mathbb{E}_{\sigma, \sigma^{\prime}} \operatorname{cov}\left(\sqrt{N} \tilde{X}_{\sigma}, \sqrt{N} \widetilde{X}_{\sigma^{\prime}}\right) & =N^{-p+1} \sum_{i_{1}, i_{2}, \ldots, i_{p}} \mathbb{E}_{\sigma, \sigma^{\prime}} \sigma_{i_{1}} \sigma_{i_{1}}^{\prime} \cdots \sigma_{i_{p}} \sigma_{i_{p}}^{\prime} \\
& =N^{-(p-2) / 2}(p-1) ! !
\end{aligned}
$$

Thus $n=1$ and the scale of fluctuations of the free energy is bigger. The failure of (1.21) happens because of sets of $p$ indices $\left\{i_{1}, \ldots, i_{p}\right\}$, where each index has a pair, as in this case $\sigma_{i_{1}} \cdots \sigma_{i_{p}}=1$ for all $\sigma$. Let us separate these sets:

$$
\begin{aligned}
\tilde{X}_{\sigma}= & N^{-p / 2} \sum_{i_{1}, i_{2}, \ldots, i_{p} \text { all in pairs }} J_{i_{1}, i_{2}, \ldots, i_{p}} \\
& +N^{-p / 2} \sum_{i_{1}, i_{2}, \ldots, i_{p} \text { not all in pairs }} J_{i_{1}, i_{2}, \ldots, i_{p}} \sigma_{i_{1}} \sigma_{i_{2}} \cdots \sigma_{i_{p}} \\
\equiv & N^{-p / 4} \sqrt{(p-1) ! !} J+X_{\sigma}^{1},
\end{aligned}
$$

where $J$ is a standard Gaussian random variable. Then

$$
\ln \frac{Z_{\beta, N}}{\mathbb{E} Z_{\beta, N}}=\beta N^{(2-p) / 4} \sqrt{(p-1) ! !} J+\ln \frac{Z_{\beta, N}^{1}}{\mathbb{E} Z_{\beta, N}},
$$

where $Z_{\beta, N}^{1}=\mathbb{E}_{\sigma} e^{\beta \sqrt{N} X_{\sigma}^{1}}$. The first term is a Gaussian random variable with mean 0 and variance $\beta^{2}(p-1)$ !! on the scale $N^{-(p-2) / 4}$. The process $X_{\sigma}^{1}$ satisfies (1.21). Hence for $X_{\sigma}^{1}$ we have $n>1$ and one can show that the fluctuations of $\ln \left(Z_{\beta, N}^{1} / \mathbb{E} Z_{\beta, N}\right)$ are on a smaller scale than $N^{-(p-2) / 4}$. This leads to the following theorem. 
THEOREM 1.4. Consider the $p$-spin SK models defined by (1.4), $p>2$, $p$ even. There exists $\beta_{p}>0$ such that, for all $\beta<\beta_{p}$,

$$
N^{(p-2) / 4} \ln \frac{Z_{\beta, N}}{\mathbb{E} Z_{\beta, N}} \stackrel{\mathcal{D}}{\rightarrow} \mathcal{N}\left(0, \beta^{2}(p-1) ! !\right) .
$$

Moreover,

$$
\inf _{0<m<1}\left(1+m^{-p}\right) \phi(m) \leq \beta_{p}^{2} \leq 2 \ln 2,
$$

where $\phi(m) \equiv[(1-m) \ln (1-m)+(1+m) \ln (1+m)] / 2$.

However, we see that for both models (1.3) and (1.4) the scale on which the partition functions fluctuate decreases rapidly as $p$ increases. One might guess that the scale becomes exponentially small in $N$ in the limiting random energy model. This is indeed true, but more surprising things happen, as the following theorem states.

THEOREM 1.5. The free energy of the REM has the following fluctuations:

(i) If $\beta<\sqrt{\ln 2 / 2}$, then

$$
\exp \left\{\frac{N}{2}\left(\ln 2-\beta^{2}\right)\right\} \ln \frac{Z_{\beta, N}}{\mathbb{E} Z_{\beta, N}} \stackrel{\mathcal{D}}{\rightarrow} \mathcal{N}(0,1) .
$$

(ii) If $\beta=\sqrt{\ln 2 / 2}$, then

$$
\sqrt{2} \exp \left\{\frac{N}{2}\left(\ln 2-\beta^{2}\right)\right\} \ln \frac{Z_{\beta, N}}{\mathbb{E} Z_{\beta, N}} \stackrel{D}{\rightarrow} \mathcal{N}(0,1) .
$$

(iii) Let $\alpha \equiv \beta / \sqrt{2 \ln 2}$. If $\sqrt{\ln 2 / 2}<\beta<\sqrt{2 \ln 2}$, then

$$
\begin{aligned}
\exp \{ & \left.\frac{N}{2}(\sqrt{2 \ln 2}-\beta)^{2}+\frac{\alpha}{2}(\ln (N \ln 2)+\ln 4 \pi)\right\} \ln \frac{Z_{\beta, N}}{\mathbb{E} Z_{\beta, N}} \\
& \stackrel{D}{\rightarrow} \int_{-\infty}^{\infty} e^{\alpha z}\left(\mathcal{P}(d z)-e^{-z} d z\right),
\end{aligned}
$$

where $\mathcal{P}$ denotes the Poisson point process on $\mathcal{R}$ with intensity measure $e^{-x} d x$.

Theorem 1.5 covers the high-temperature regime. However, in the REM we can also compute the fluctuations in the low-temperature phase.

THEOREM 1.6. Let $\alpha \equiv \beta / \sqrt{2 \ln 2}$.

(i) If $\beta=\sqrt{2 \ln 2}$, then

$$
\begin{gathered}
e^{(\ln (N \ln 2)+\ln 4 \pi) / 2}\left(\frac{Z_{\beta, N}}{\mathbb{E} Z_{\beta, N}}-\frac{1}{2}+\frac{\ln (N \ln 2)+\ln 4 \pi}{4 \sqrt{\pi N \ln 2}}\right) \\
\stackrel{\mathscr{D}}{\rightarrow} \int_{-\infty}^{0} e^{\alpha z}\left(\mathcal{P}(d z)-e^{-z} d z\right)+\int_{0}^{\infty} e^{z} \mathcal{P}(d z) .
\end{gathered}
$$


(ii) If $\beta>\sqrt{2 \ln 2}$, then

$$
\begin{aligned}
& \exp \left\{-N(\beta \sqrt{2 \ln 2}-\ln 2)+\frac{\alpha}{2}(\ln (N \ln 2)+\ln 4 \pi)\right\} Z_{\beta, N} \\
& \quad \stackrel{D}{\rightarrow} \int_{-\infty}^{\infty} e^{\alpha z} \mathcal{P}(d z)
\end{aligned}
$$

and

$$
\ln Z_{\beta, N}-\mathbb{E} \ln Z_{\beta, N} \stackrel{\mathcal{D}}{\rightarrow} \ln \int_{-\infty}^{\infty} e^{\alpha z} \mathcal{P}(d z)-\mathbb{E} \ln \int_{-\infty}^{\infty} e^{\alpha z} \mathcal{P}(d z)
$$

REMARK. Note that expressions like $\int_{-\infty}^{0} e^{\alpha z}\left(\mathcal{P}(d z)-e^{-z} d z\right)$ are always understood as $\lim _{y \downarrow-\infty} \int_{y}^{0} e^{\alpha z}\left(\mathcal{P}(d z)-e^{-z} d z\right)$. We will see that all the functionals of the Poisson point process appearing are almost surely finite random variables.

REMARK. Note that the Poisson integral $\int_{-\infty}^{\infty} e^{\alpha z} \mathcal{P}(d z)$ is the partition function of Ruelle's version of the REM [23]. Thus (1.32) affirms that, above the critical temperature, the fluctuations of the free energy of the REM converge in distribution to those of Ruelle's model. It is obvious that Ruelle must have been aware of this fact when writing his paper, but he did not give a proof. Rigorous results in this context have been obtained by Galvez, Martinez and Picco [14]. It is interesting to compare them to our result. In our notation, their Theorem 1 asserts that, for $\beta \geq \sqrt{2 \ln 2}$,

$$
\begin{aligned}
& \limsup _{N \uparrow \infty} \frac{\ln \left[Z_{\beta, N} e^{-N[\beta \sqrt{2 \ln 2}-\ln 2]}\right]}{\alpha \ln N}=+\frac{1}{2} \quad \text { a.s., } \\
& \liminf _{N \uparrow \infty} \frac{\ln \left[Z_{\beta, N} e^{-N[\beta \sqrt{2 \ln 2}-\ln 2]}\right]}{\alpha \ln N}=-\frac{1}{2} \quad \text { a.s. }
\end{aligned}
$$

Moreover, they explained that, for $\beta>\sqrt{2 \ln 2}$,

$$
\frac{\ln \left[Z_{\beta, N} e^{-N[\beta \sqrt{2 \ln 2}-\ln 2]}\right]}{\alpha \ln N} \rightarrow-\frac{1}{2} \quad \text { in probability. }
$$

If we denote the logarithm of the left-hand side of (1.31) by $G_{N}$, we see that

$$
\frac{\ln \left[Z_{\beta, N} e^{-N[\beta \sqrt{2 \ln 2}-\ln 2]}\right]}{\alpha \ln N}=-\frac{1}{2}+\frac{G_{N}}{\alpha \ln N}-\frac{1}{2} \frac{\ln (4 \pi \ln 2)}{\ln N} .
$$

This explains immediately why this quantity converges to $-1 / 2$ in probability. Also, by the positivity of $G_{N}$, it gives that the liminf is $-1 / 2$ a.s. almost immediately. Thus we see that (1.31) is the weak limit theorem to which Theorem 1 of [14] is the "law of the (noniterated) logarithm." In their Theorem 2 Galvez, Martinez and Picco observed that the process of Boltzmann weights 
$\exp \left(-\beta \sqrt{N} X_{\sigma}\right)$ properly rescaled converges to the Poisson process introduced by Ruelle. As these authors state, this is a direct consequence of their Lemma 4, which in turn is a classical result of extreme value theory (see, e.g., [17], Theorem 5.7.2). Galvez, Martinez and Picco did not, however, discuss whether and when this implies the convergence of the normalized partition function, a fact which is not obvious and indeed only holds if $\beta>\sqrt{2 \ln 2}$. Thus the gap remaining to fully establish the connection between the REM and Ruelle's model is now filled.

REMARK. It is interesting to observe that in the REM there is a second "phase transition" within the high-temperature phase at which the fluctuations become non-Gaussian. In fact, in the REM the main phase transition can be interpreted as a breakdown of the law of large numbers, while the second transition corresponds to a breakdown of the central limit theorem.

The remainder of this paper is organized as follows. In the next section we present the proofs of Theorems 1.2-1.4. The proof of Theorems 1.2 and 1.3 is based on an adaptation of the martingale method of Comets and Neveu. The essential new ingredient is the rather involved truncation procedure inspired by Talagrand's work. However, in the proof of the CLT, the computational aspects become even more involved and require the consideration of truncated third moments of the partition function. For this reason Section 2 is rather long and quite technical. However, the proof is organized in such a way that the CLT is first proven for "very high" temperatures where no truncations are necessary, while the more technical aspects needed to approach the critical temperature are dealt with separately later. The proof of Theorem 1.4 is much simpler and does not use the martingale method, but only truncation. Section 3 is devoted to proving Theorems 1.5 and 1.6 for the REM. It is technically completely different and independent from Section 2. It can therefore be read independently from the rest of the paper. In Appendix A we discuss the case of more general covariance functions and explain the scalings and the CLT's that should be expected.

\section{The CLT in the $p$-spin model.}

PROOF OF THEOREMS 1.2 AND 1.3. The proof of the central limit theorem in the $p$-spin SK model (1.3) relies on a martingale central limit theorem, which uses the fact that a Gaussian random variable can always be seen as the marginal distribution of a Brownian motion. Thus we follow Comets and Neveu and introduce the $p$-parameter family of independent standard Brownian motions $\left(J_{i_{1}, i_{2}, \ldots, i_{p}}(t), t \in \mathbb{R}^{+}\right)_{i_{1}, i_{2}, \ldots, i_{p} \in \mathbb{N}}$ with $\mathbb{E} J_{i_{1}, i_{2}, \ldots, i_{p}}(t)=0$ and $\mathbb{E} J_{i_{1}, i_{2}, \ldots, i_{p}}^{2}(t)=t$. The Hamiltonian of the $p$-spin SK model can then be written as $H_{N}(\sigma, t)=$ $\sqrt{N} X_{\sigma}(t)$, where

$$
X_{\sigma}(t)=\left(\begin{array}{c}
N \\
p
\end{array}\right)^{-1 / 2} \sum_{1 \leq i_{1}<i_{2}<\cdots<i_{p} \leq N} J_{i_{1}, i_{2}, \ldots, i_{p}}(t) \sigma_{i_{1}} \sigma_{i_{2}} \cdots \sigma_{i_{p}} .
$$


Note that we can also consider it as a Gaussian process on $\{-1,1\}^{N} \times \mathbb{R}^{+}$with mean 0 and correlation function

$$
\begin{aligned}
& \operatorname{cov}\left(X_{\sigma}(t), X_{\sigma^{\prime}}(s)\right) \\
& \quad=\frac{(s \wedge t)}{N(N-1) \cdots(N-p+1)} \sum_{i_{1}, i_{2}, \ldots, i_{p} \text { different }} \sigma_{i_{1}} \sigma_{i_{1}}^{\prime} \cdots \sigma_{i_{p}} \sigma_{i_{p}}^{\prime} .
\end{aligned}
$$

In particular, we have $\mathbb{E} H_{N}^{2}(\sigma, t)=N t$ and $\mathbb{E} \exp \left\{H_{N}(t, \sigma)\right\}=\exp \{N t / 2\}$ for all $\sigma$.

The following proposition provides a representation of the covariances of the Hamiltonian as a polynomial function of the overlap $\sigma \cdot \sigma^{\prime} \equiv \sum_{i=1}^{N} \sigma_{i} \sigma_{i}^{\prime}$. We will not need the explicit form of the coefficients in the rest of the paper but their order.

PROPOSITION 2.1. There exist functions $\gamma_{p-2 k}(N), k=0,1, \ldots,[p / 2]$, such that, for all spin configurations $\sigma, \sigma^{\prime} \in \Sigma_{N}$,

$$
\sum_{i_{1}, i_{2}, \ldots, i_{p} \text { different }} \sigma_{i_{1}} \sigma_{i_{1}}^{\prime} \cdots \sigma_{i_{p}} \sigma_{i_{p}}^{\prime}=\sum_{k=0}^{[p / 2]} \gamma_{p-2 k}(N)\left(\sigma \cdot \sigma^{\prime}\right)^{p-2 k} .
$$

Moreover, $\gamma_{p}(N)=1$ and $\gamma_{p-2 k}(N)=N^{k} d_{p-2 k}(1+O(1 / N))$ as $N \uparrow \infty$, where

$$
\begin{aligned}
d_{p-2 k} & =(-1)^{k} \frac{p(p-1) \cdots(p-2 k+1)}{2^{k} k !} \\
& =(-1)^{k}\left(\begin{array}{c}
p \\
2 k
\end{array}\right)(k-1) ! !, \quad 0<k \leq \frac{p}{2} .
\end{aligned}
$$

PROOF. Let us write

$$
\begin{aligned}
\sum_{i_{1}, i_{2}, \ldots, i_{p} \text { different }} \sigma_{i_{1}} \sigma_{i_{1}}^{\prime} \cdots \sigma_{i_{p}} \sigma_{i_{p}}^{\prime} & =\sum_{i_{1}, i_{2}, \ldots, i_{p}} \prod_{\{l, k\}} \mathbb{1}_{i_{k} \neq i_{l}} \sigma_{i_{1}} \sigma_{i_{1}}^{\prime} \cdots \sigma_{i_{p}} \sigma_{i_{p}}^{\prime} \\
& =\sum_{i_{1}, i_{2}, \ldots, i_{p}} \prod_{\{l, k\}}\left(1-\mathbb{1}_{i_{k}=i_{l}}\right) \sigma_{i_{1}} \sigma_{i_{1}}^{\prime} \cdots \sigma_{i_{p}} \sigma_{i_{p}}^{\prime} .
\end{aligned}
$$

Now we can expand the product

$$
\prod_{\{l, k\}}\left(1-\mathbb{1}_{i_{k}=i_{l}}\right)=\sum_{s=1}^{[p(p-1) / 2]}(-1)^{s} \sum_{\left(l_{1}, k_{1}\right), \ldots,\left(l_{s}, k_{s}\right)} \prod_{r=1}^{s} \mathbb{1}_{i_{l_{r}}=i_{k_{r}}},
$$

where the last sum is over all distinct pairs of indices taking values in $\{1, \ldots, p\}$. Now consider the set of bonds $\left(l_{i}, k_{i}\right)$ as a graph on $\{1, \ldots, p\}$. This graph induces a connectivity structure on the set $\mathcal{P} \equiv\{1, \ldots, p\}$ and we can decompose $\mathcal{P}$ into its connected components, $\mathcal{C}_{1}, \ldots, \mathcal{C}_{r}$. Each contribution in the sum in (2.5) that gives rise to the same decomposition into connected components will yield the 
same contribution (up to a sign) to the covariance function when (2.5) is inserted into (2.4), namely,

$$
\sum_{1 \leq j_{1}, \ldots, j_{r} \leq N} \prod_{l=1}^{r}\left(\sigma_{j_{l}} \sigma_{j_{l}}^{\prime}\right)^{\left|\mathcal{C}_{l}\right|}
$$

Thus, if we denote by $n_{\text {odd }}$ the number of connected components of odd size and by $n_{\text {even }}$ the number of even size, this is equal to

$$
N^{n_{\text {even }}} N^{n_{\text {odd }}} R_{N}\left(\sigma, \sigma^{\prime}\right)^{n_{\text {odd }}}=N^{r} R_{N}\left(\sigma, \sigma^{\prime}\right)^{n_{\text {odd }}} .
$$

Note that $n_{\text {odd }}$ differs from $p$ always by an even number, and so all graphs that give rise to a partition with $n_{\text {odd }}=p-2 k$ contribute to the coefficient $\gamma_{p-2 k}(N)$. Due to (2.7), the largest contribution (in powers of $N$ ) comes from those graphs that provide a decomposition with maximal number of connected components, given the number of odd-sized components. Obviously, in such a decomposition all evensized components are of size 2 and all odd-sized components are of size 1; that is, there are precisely $k$ even-sized components. To such a decomposition corresponds a unique graph consisting of $k$ mutually disjoint bonds (pairs). The number of ways to select $k$ mutually disjoint pairs from $p$ objects is $\left(\begin{array}{c}p \\ 2 k\end{array}\right)(k-1)$ !!. This proves the proposition.

It follows from this proposition that

$$
\operatorname{cov}\left(H_{N}(\sigma, t), H_{N}\left(\sigma^{\prime}, s\right)\right)=N(s \wedge t) f_{N}^{p}\left(R_{N}\left(\sigma, \sigma^{\prime}\right)\right),
$$

where $R_{N}\left(\sigma, \sigma^{\prime}\right)=\left(\sigma \cdot \sigma^{\prime}\right) N^{-1}$ and

$$
f_{N}^{p}(x)=\sum_{k=0}^{p / 2} d_{p-2 k} N^{-k} x^{p-2 k}(1+O(1 / N))
$$

as $N \uparrow \infty$. We will often use the expansion

$$
f_{N}^{p}(x)=(1+O(1 / N)) x^{p}+O(1 / N) x^{p-2}+O\left(1 / N^{2}\right), \quad N \uparrow \infty,
$$

where $O(1)$ is uniform for $x \in[0,1]$. To conclude the discussion of the covariances of the Hamiltonian, let us note the following important properties:

$$
\begin{aligned}
\mathbb{E}_{\sigma, \sigma^{\prime}} \operatorname{cov}\left(H_{N}(\sigma, t), H_{N}\left(\sigma^{\prime}, s\right)\right) \\
=(s \wedge t) N \mathbb{E}_{\sigma, \sigma^{\prime}} f_{N}^{p}\left(R_{N}\left(\sigma, \sigma^{\prime}\right)\right) \\
=(s \wedge t) N\left(\begin{array}{c}
N \\
p
\end{array}\right)^{-1} \sum_{i_{1}<i_{2}<\cdots<i_{p}} \mathbb{E}_{\sigma, \sigma^{\prime}} \sigma_{i_{1}} \sigma_{i_{1}}^{\prime} \cdots \sigma_{i_{p}} \sigma_{i_{p}}^{\prime}=0
\end{aligned}
$$


and

$$
\begin{aligned}
\mathbb{E}_{\sigma, \sigma^{\prime}} & \left(\operatorname{cov}\left(H_{N}(\sigma, t), H_{N}\left(\sigma^{\prime}, s\right)\right)\right)^{2} \\
= & \left(s^{2} \wedge t^{2}\right) N^{2} \mathbb{E}_{\sigma, \sigma^{\prime}}\left(f_{N}^{p}\left(R_{N}\left(\sigma, \sigma^{\prime}\right)\right)\right)^{2} \\
= & \left(s^{2} \wedge t^{2}\right) N^{2}\left(\begin{array}{c}
N \\
p
\end{array}\right)^{-2} \mathbb{E}_{\sigma, \sigma^{\prime}}\left(\sum_{i_{1}<i_{2}<\cdots<i_{p}} \sigma_{i_{1}} \sigma_{i_{1}}^{\prime} \cdots \sigma_{i_{p}} \sigma_{i_{p}}^{\prime}\right)^{2} \\
= & \left(s^{2} \wedge t^{2}\right) N^{2}\left(\begin{array}{c}
N \\
p
\end{array}\right)^{-1}(1+O(1 / N)) .
\end{aligned}
$$

For later convenience we introduce the normalized partition function

$$
\bar{Z}_{N}(t)=\mathbb{E}_{\sigma} \exp \left\{H_{N}(t, \sigma)-N t / 2\right\} .
$$

It is related to the partition function $Z_{\beta, N}$ of Section 1 by $\bar{Z}_{N}\left(\beta^{2}\right)=Z_{\beta, N} / \mathbb{E} Z_{\beta, N}$, with equality holding in law. The important point of this construction is that, for all fixed $N>1, \bar{Z}_{N}(t)$ is a continuous martingale in the variable $t$ with $\mathbb{E} \bar{Z}_{N}(t)=1$.

We begin the proof with some preliminary steps along the lines of [9]. Let us find the bracket $\left\langle\bar{Z}_{N}(t)\right\rangle$ of the martingale $\bar{Z}_{N}(t)$, that is, the unique increasing process vanishing at 0 , such that $\bar{Z}_{N}^{2}(t)-\left\langle\bar{Z}_{N}(t)\right\rangle$ is a continuous martingale (see [22]). By Itô's formula, $\bar{Z}_{N}(t)$ satisfies the following stochastic differential equation:

$$
d \bar{Z}_{N}(t)=\mathbb{E}_{\sigma} \exp \left\{H_{N}(t, \sigma)-N t / 2\right\} d H_{N}(t, \sigma) .
$$

Then, due to the well-known properties of martingale brackets,

$$
\begin{aligned}
\left\langle\bar{Z}_{N}(t)\right\rangle & =\mathbb{E}_{\sigma, \sigma^{\prime}}\left\langle\int_{0}^{t} e^{H_{N}(s, \sigma)-N s / 2} d H_{N}(s, \sigma), \int_{0}^{t} e^{H_{N}\left(s, \sigma^{\prime}\right)-N s / 2} d H_{N}\left(s, \sigma^{\prime}\right)\right\rangle \\
& =\mathbb{E}_{\sigma, \sigma^{\prime}} \int_{0}^{t} e^{H_{N}(s, \sigma)+H_{N}\left(s, \sigma^{\prime}\right)-N s} N f_{N}^{p}\left(R_{N}\left(\sigma, \sigma^{\prime}\right)\right) d s .
\end{aligned}
$$

Since

$$
\begin{aligned}
\mathbb{E} \int_{0}^{t} \bar{Z}_{N}^{-2}(s) d\left\langle\bar{Z}_{N}(s)\right\rangle & \\
\quad= & \mathbb{E} \int_{0}^{t} \frac{\mathbb{E}_{\sigma, \sigma^{\prime}} e^{H_{N}(s, \sigma)+H_{N}\left(s, \sigma^{\prime}\right)-N s} N f_{N}^{p}\left(R_{N}\left(\sigma, \sigma^{\prime}\right)\right)}{\mathbb{E}_{\sigma, \sigma^{\prime}} e^{H_{N}(s, \sigma)+H_{N}\left(s, \sigma^{\prime}\right)-N s}} d s \leq N t<\infty,
\end{aligned}
$$

we may introduce the continuous local martingale $M_{N}(t)=\int_{0}^{t} \bar{Z}_{N}^{-1}(s) d \bar{Z}_{N}(s)$. Thus $\bar{Z}_{N}(t)$ solves the stochastic differential equation

$$
d \bar{Z}_{N}(t)=\bar{Z}_{N}(t) d M_{N}(t)
$$

and the following fundamental representation of $\bar{Z}_{N}(t)$ holds:

$$
\bar{Z}_{N}(t)=\exp \left\{M_{N}(t)-\frac{1}{2}\left\langle M_{N}(t)\right\rangle\right\},
$$


which can be rewritten conveniently as

$$
N^{(p-2) / 2} \ln \bar{Z}_{N}(t)=N^{(p-2) / 2} M_{N}(t)-\frac{1}{2} N^{(p-2) / 2}\left\langle M_{N}(t)\right\rangle .
$$

Here $\left\langle M_{N}(t)\right\rangle$ is the bracket of $M_{N}(t)$ and $\left\langle M_{N}(t)\right\rangle=\int_{0}^{t} \bar{Z}_{N}^{-2}(s) d\left\langle\bar{Z}_{N}(s)\right\rangle$. Let us note that

$$
\begin{aligned}
\frac{d}{d t}\left\langle M_{N}(t)\right\rangle & =\bar{Z}_{N}^{-2}(t) \frac{d}{d t}\left\langle\bar{Z}_{N}(t)\right\rangle \\
& =\bar{Z}_{N}^{-2}(t)\left(\mathbb{E}_{\sigma, \sigma^{\prime}} e^{H_{N}(t, \sigma)+H_{N}\left(t, \sigma^{\prime}\right)-N t} N f_{N}^{p}\left(R_{N}\left(\sigma, \sigma^{\prime}\right)\right)\right) .
\end{aligned}
$$

Note also that $M_{N}(t)$ is locally square integrable. In fact, by (2.15),

$$
\mathbb{E} M_{N}^{2}(t)=\mathbb{E}\left\langle M_{N}(t)\right\rangle=\mathbb{E} \int_{0}^{t} \bar{Z}_{N}^{-2}(s) d\left\langle\bar{Z}_{N}(s)\right\rangle \leq N t<\infty .
$$

To prove Theorems 1.2 and 1.3, we will show that, for all $t$ satisfying

$$
t<\inf _{m_{1}, m_{2}, m_{3} \in \mathcal{A}} Y_{p}\left(m_{1}, m_{2}, m_{3}\right),
$$

the bracket of the local martingale $N^{(p-2) / 2} M_{N}(t)$, which is $N^{p-2}\left\langle M_{N}(t)\right\rangle$, converges to $t^{2} p ! / 2$ in probability as $N \uparrow+\infty$. Then, by the martingale convergence theorem (see Theorem 3.1.8 in [16]), the first term in (2.17), the local martingale $N^{(p-2) / 2} M_{N}(t)$, converges in law to a centered Gaussian process with independent increments $M_{\infty}(t)$ as $N \rightarrow \infty$ and $\mathbb{E}\left(M_{\infty}(t)\right.$ $\left.M_{\infty}(s)\right)^{2}=\left(t^{2}-s^{2}\right) p ! / 2$. Moreover, if $p>2$, the second term in (2.17), $N^{-(p-2) / 2}\left\langle N^{(p-2) / 2} M_{N}(t)\right\rangle$, converges to 0 . These two facts together with the equality in law $Z_{N, \beta} / \mathbb{E} Z_{N, \beta}=\bar{Z}_{N}\left(\beta^{2}\right)$ imply immediately the statement of Theorem 1.2. Note that, in the case $p=2$, that is, the SK model considered in [9], the second term in (2.17) does not vanish, but converges to one half of the variance of the process $M_{\infty}(t)$. Therefore, in the case $p=2$, the fluctuations of the free energy are not centered in contrast to the case $p>2$.

SKETCH OF THE PROOF OF THEOREMS 1.2 AND 1.3. We will now outline further steps of the proof. First, we show the convergence $N^{p-2}\left\langle M_{N}(t)\right\rangle \rightarrow$ $t^{2} p ! / 2$ on a more restricted interval of $t$. Lemma 2.2 reduces this problem to the convergence of

$$
N^{p-2} \mathbb{E}\left|V_{N}(t)\right| \rightarrow 0 \quad \text { as } N \uparrow+\infty,
$$

where

$$
V_{N}(t):=N^{-(p-2)} \mathbb{E}_{\sigma, \sigma^{\prime}}\left(N^{p-1} f_{N}^{p}\left(R_{N}\left(\sigma, \sigma^{\prime}\right)\right)-t p !\right) e^{H_{N}(t, \sigma)+H_{N}\left(t, \sigma^{\prime}\right)-N t} .
$$

The proof of this lemma is based on the fact that

$$
N^{p-2} \frac{d}{d t}\left\langle M_{N}(t)\right\rangle-t p !=N^{p-2} \frac{V_{N}(t)}{\bar{Z}_{N}^{2}(t)},
$$


and is performed via integration. It almost mimics the proof proposed in [9]. In particular, we use the fact that $\bar{Z}_{N}^{2}(t)$ is not small on events of large probability. The convergence (2.21) is proved in Proposition 2.3. Let us give some intuition for it. One can write

$$
\mathbb{E} V_{N}(t)=\sum_{m=0, \pm 1 / N, \ldots, \pm 1}\left(N f_{N}^{p}(m)-N^{2-p} t p !\right) e^{t N f_{N}^{p}(m)} \mathbb{P}_{m}
$$

where by Stirling's formula

$$
\mathbb{P}_{m} \equiv \mathbb{P}_{\sigma, \sigma^{\prime}}\left(\sigma \cdot \sigma^{\prime}=m N\right) \sim \frac{2}{\sqrt{2 \pi(1+m)(1-m) N}} e^{-N \phi(m)},
$$

$\phi(m)=[(1+m) \ln (1+m)+(1-m) \ln (1-m)] / 2$ (here and in the rest of the paper we use the symbol $\sim$ to denote asymptotic equivalence, i.e., $a_{N} \sim$ $\left.b_{N} \Leftrightarrow \lim _{N \uparrow+\infty} \frac{a_{N}}{b_{N}}=1\right)$. Note that $\phi(m)=m^{2} / 2(1+o(1))$ as $m \rightarrow 0$. Now split the right-hand side of (2.23) into two terms: the summation in the first term will be over $m$ with $|m|$ small enough (namely, $|m|<N^{-\alpha}$ with an arbitrary $1 / 3<\alpha<1 / 2$ ), and in the second, over all other $m$. It is not difficult to treat the first term. We replace $e^{t N f_{N}^{p}(m)}$ by its Taylor expansion and respresent this term as the sum of three:

$$
\begin{aligned}
& \sum_{|m| \text { small }} N f_{N}^{p}(m)\left(1-N^{2-p} t^{2} p !\right) \mathbb{P}_{m} \\
& \quad+\sum_{|m| \text { small }}\left(t N^{2}\left(f_{N}^{p}(m)\right)^{2}-t N^{2-p} p !\right) \mathbb{P}_{m} \\
& \quad+\sum_{|m| \text { small }}\left(N f_{N}^{p}(m)-N^{2-p} t p !\right) O\left(\left(t N f_{N}^{p}(m)\right)^{2}\right) \mathbb{P}_{m}
\end{aligned}
$$

The first of these three goes to 0 exponentially fast. This follows from the property of the covariances (2.10) and from an exponentially small order of the probability (2.24) on the sets of $m$ distant from 0 for at least $N^{-\alpha}$ with $\alpha<1 / 2$. The second term of (2.25) converges to 0 on the scale $N^{2-p}$. This is a consequence of the second property of the covariances $\mathbb{E}_{\sigma, \sigma^{\prime}}\left(f_{N}^{p}\left(R_{N}\left(\sigma, \sigma^{\prime}\right)\right)\right)^{2} \sim$ $N^{-p} p$ ! (2.11) and of the same argument about the concentration of $P_{m}$ in 0 . To analyze the third term of (2.25), one has, by Proposition $2.1, t N f_{N}^{p}(m)=$ $O\left(N^{1-p \alpha}\right)=o(1)$ for $m=O\left(N^{-\alpha}\right)$ with $\alpha>1 / 3, p \geq 3$. Let us substitute the expansion $\phi(m) \sim m^{2} / 2$ and put $s=m \sqrt{N}$ in this term. Again, by Proposition 2.1,

$$
N^{p / 2} f_{N}^{p}(m)=\sum_{k=0}^{[p / 2]} d_{p-2 k}(m \sqrt{N})^{p-2 k}(1+O(1 / N)) .
$$


Then the third term of (2.25) can be estimated up to some constant factor and terms of smaller order by

$$
\begin{aligned}
& \sum_{|m| \text { small }}\left|N^{3} t^{2}\left(f_{N}^{p}(m)\right)^{3}\right|+\left|N^{4-p} t^{3} p !\left(f_{N}^{p}(m)\right)^{2}\right| \mathbb{P}_{m} \\
& \quad \frac{N^{3-3 p / 2}}{\sqrt{2 \pi N}} \sum_{m \sqrt{N}=s}\left(t^{2}\left|D_{p}(s)\right|^{3}+N^{1-p / 2} t^{3} p !\left|D_{p}(s)\right|^{2}\right) e^{-s^{2} / 2},
\end{aligned}
$$

where $D_{p}(s) \equiv \sum_{k=0}^{[p / 2]} d_{p-2 k} s^{p-2 k}$. The sum (2.26) is the integral sum of $\left|D_{p}(s)\right|^{3}$ over the Gaussian density, which tends to the corresponding finite integral. Therefore the scale of fluctuations of the third part of (2.25) is $N^{3-3 p / 2}$ smaller than $N^{2-p}$ for $p \geq 3$. [Another straightforward but not very elegant way of considering this term would be to estimate the left-hand side of (2.26) directly as $O\left(N^{3-3 p \alpha}\right)+O\left(N^{4-p-2 p \alpha}\right)$ and to fix $4 / 9<\alpha<1 / 2$ to make its scale smaller than $N^{2-p}$ for all $p \geq 3$.]

To ensure the convergence to 0 of the second term of $\mathbb{E} V_{N}(t)$ (the one with correlations $|m|$ not close to 0 ), the power of the exponent in it, which is $N\left(t f_{N}^{p}(m)-\phi(m)\right)=N\left(t m^{p}-\phi(m)\right)+O(1)$, should be negative. Thus, for all $t<\inf _{0<m<1} \phi(m) m^{-p}$, we get $N^{p-2} \mathbb{E} V_{N}(t) \rightarrow 0$. Note that Proposition 2.3 states a stronger result, namely, (2.21). To get rid of the absolute value of $V_{N}(t)$ in (2.21), we follow an idea suggested in [9] to apply the Cauchy-Schwarz inequality. Thus, instead of $\mathbb{E}\left|V_{N}(t)\right|$, we get $W_{N}(t)$ (see the proof of Proposition 2.3), which refers to the third moment of $\bar{Z}_{N}(t)$. This will make technical computations slightly tougher and will lead to the bound on $t$ given in Lemma 2.2.

Note also that these arguments are valid only for $p \geq 3$ : in the case $p=2$, the third part of (2.25) does not vanish at the right scale. The case $p=2$ of [9] and [28] is different, since, there, the correlation function $t f_{N}^{2}(m) \sim \mathrm{tm}^{2}$ and the entropy $\phi(m) \sim m^{2} / 2$ are in "competition" in the exponent $e^{N\left(t f_{N}^{2}(m)-\phi(m)\right)}$, while in our case $p>2$ the entropy dominates. The case $p=2$ is treated in [9] by the multidimensional central limit theorem for $N$ independent vectors $\left(\sigma_{i} \sigma_{i}^{\prime}, \sigma_{i}^{\prime} \sigma_{i}^{\prime \prime}, \sigma_{i} \sigma_{i}^{\prime \prime}\right)$.

Next, we will extend the bound (2.32) to the full regime announced in (2.20). We have seen that (2.32) was imposed by configurations of spins with rather big correlations $m$ in the sum (2.23). We will reduce their contribution, using Talagrand's idea to truncate the Hamiltonian. Consider, instead of $V_{N}(t)$,

$$
\begin{aligned}
\tilde{V}_{N}(t, \varepsilon)= & \mathbb{E}_{\sigma, \sigma^{\prime}}\left(N f_{N}^{p}\left(R_{N}\left(\sigma, \sigma^{\prime}\right)\right)-N^{2-p} t p !\right) e^{H_{N}(t, \sigma)+H_{N}\left(t, \sigma^{\prime}\right)-N t} \\
& \times \mathbb{1}_{\left\{H_{N}(t, \sigma)<(1+\varepsilon) t N, H_{N}\left(t, \sigma^{\prime}\right)<(1+\varepsilon) t N\right\}}
\end{aligned}
$$

for some $\varepsilon>0$. Then

$$
\begin{aligned}
\mathbb{E} \tilde{V}_{N}(t, \varepsilon)= & \sum_{m=0, \pm 1 / N, \ldots, \pm 1}\left(N f_{N}^{p}(m)-t N^{2-p} p !\right) \mathbb{P}_{m} \\
& \times \mathbb{E} e^{\sqrt{N t} \xi_{1}+\sqrt{N t} \xi_{2}-N t} \mathbb{1}_{\left\{\xi_{1}<\sqrt{N t}(1+\varepsilon), \xi_{2}<\sqrt{N t}(1+\varepsilon)\right\}},
\end{aligned}
$$


where $\xi_{1}, \xi_{2}$ are standard Gaussians with $\operatorname{cov}\left(\xi_{1}, \xi_{2}\right)=f_{N}^{p}(m)$. Let us again split $\mathbb{E} \widetilde{V}_{N}(t, \varepsilon)$ into two terms with "small" and "large" $m$ in the sum (2.27). The analysis of the first term is completely analogous to that in the case of $V_{N}(t)$. We can neglect the truncation here, since $\xi_{1}$ and $\xi_{2}$ are almost independent. In the second term, $\xi_{1}$ and $\xi_{2}$ are more correlated. But, due to the truncation, the expectation of the exponent involved in this term is much smaller than $e^{t N f_{N}^{p}(m)}$. In fact, by the elementary estimate (B.2) for Gaussian random variables,

$$
\begin{aligned}
\mathbb{E} \exp & \left\{\sqrt{N t} \xi_{1}+\sqrt{N t} \xi_{2}-N t\right\} \mathbb{1}_{\left\{\xi_{1}<\sqrt{N t}(1+\varepsilon), \xi_{2}<\sqrt{N t}(1+\varepsilon)\right\}} \\
& \leq \mathbb{E} \exp \left\{\sqrt{N t\left(2+2 f_{N}^{p}(m)\right)} \xi-N t\right\} \mathbb{1}_{\left\{\xi<2 \sqrt{N t}(1+\varepsilon)\left(2+2 f_{N}^{p}(m)\right)^{-1 / 2}\right\}} \\
& \leq \exp \left\{\left[-4 N t(1+\varepsilon)^{2}\right]\left[4+4 f_{N}^{p}(m)\right]^{-1}+2 N t(1+\varepsilon)-N t\right\} \\
& =\exp \left\{N t\left[f_{N}^{p}(m)(1+2 \varepsilon)-\varepsilon^{2}\right]\left[1+f_{N}^{p}(m)\right]^{-1}\right\} .
\end{aligned}
$$

Then, by (2.9) for any

$$
t<\inf _{0<m<1}\left(1+m^{-p}\right) \phi(m)
$$

and for an appropriate choice of $\varepsilon$, all terms of the sum (2.27) with $m$ not close to 0 are exponentially small. This implies $N^{p-2} \mathbb{E} \widetilde{V}_{N}(t, \varepsilon) \rightarrow 0$. The bound (2.29) is Talagrand's bound for the critical temperature in the $p$-spin SK model; see (1.8). It tends to $2 \ln 2$ as $p \uparrow+\infty$.

To incorporate this idea into our proof, we reduce the problem of convergence $N^{p-2}\left\langle M_{N}(t)\right\rangle \rightarrow t^{2} p ! / 2$ to the following statements:

$$
N^{p-2} \mathbb{E}\left|\widetilde{V}_{N}(t, \varepsilon)\right| \rightarrow 0
$$

and

$$
N^{p-2} \mathbb{E}\left|\left(V_{N}(t)-\widetilde{V}_{N}(t, \varepsilon)\right) \bar{Z}_{N}^{-2}(t)\right| \rightarrow 0
$$

for all $\varepsilon>0$. This is derived in Lemma 2.4 again from (2.21). In Proposition 2.5 we show (2.30). Note that if the absolute value were in (2.17) we would have obtained our CLT just up to Talagrand's bound for the critical temperature (2.16). But again, because of the absolute value, we must apply the Cauchy-Schwarz inequality and pass to the third moment of $\bar{Z}_{N}(t)$. This makes technical computations much harder and leads to a different condition on $\beta$, whose large $p$ asymptotics will, however, be seen to be the same (up to constants) as that of Talagrand's bound. Namely, we get three standard Gaussian random variables $\xi_{1}, \xi_{2}, \xi_{3}$ with covariances $f_{N}^{p}\left(m_{1}\right), f_{N}^{p}\left(m_{2}\right), f_{N}^{p}\left(m_{3}\right)$. To benefit from the truncation for obtaining a good bound on $t$, we have to take into account four different cases: one when all $m_{1}, m_{2}, m_{3}$ are large and others when two of these correlations 
are large and the third is small. Then the analogue of (2.29) is the minimum of four estimates of this kind. Therefore the bound (2.20) is the minimum of four functions. The convergence (2.31) is the subject of Proposition 2.6. Its proof uses ideas of Talagrand and a concentration of measure inequality.

LEMMA 2.2. Let

$$
T<\inf _{\mathcal{A}} \frac{I\left(m_{1}, m_{2}, m_{3}\right)}{m_{1}^{p}+m_{2}^{p}+m_{3}^{p}}
$$

Then

$$
\sup _{0 \leq t \leq T}\left|N^{p-2}\left\langle M_{N}(t)\right\rangle-t^{2} p ! / 2\right| \rightarrow 0
$$

in probability.

ProOF. Let us denote by

$$
V_{N}(t)=\frac{d}{d t}\left\langle\bar{Z}_{N}(t)\right\rangle-N^{2-p} t \bar{Z}_{N}^{2}(t) p !
$$

Then

$$
\begin{aligned}
\frac{d}{d t} N^{p-2}\left\langle M_{N}(t)\right\rangle-t p ! & =N^{p-2} V_{N}(t) \bar{Z}_{N}^{-2}(t) \\
& =N^{p-2} V_{N}(t) \exp \left\{-2 M_{N}(t)+\left\langle M_{N}(t)\right\rangle\right\} .
\end{aligned}
$$

Let us introduce the events $A_{a, b}^{N}:=\left\{-M_{N}(t) \leq a+(b / 2)\left\langle M_{N}(t)\right\rangle\right.$ for all $\left.t \geq 0\right\}$. Note that, by an appropriate choice of $a>0$ and $b>0$, their probabilities can be made arbitrarily close to 1 . In fact, the process $B_{N}(t)=M_{N}\left(S_{t}\right)$, where $S_{t}=$ $\min \left\{s \mid\left\langle M_{N}(s)\right\rangle=t\right\}$, is a standard Brownian motion and $M_{N}(t)=B_{N}\left(\left\langle M_{N}(t)\right\rangle\right)$. By the well-known fact for Brownian motion,

$$
\mathbb{P}\left\{A_{a, b}^{N}\right\}=\mathbb{P}\left\{-B_{N}(t) \leq a+(b / 2) t \text { for all } t \geq 0\right\} \geq 1-\exp \{-a b\} .
$$

We have

$$
\begin{aligned}
& \left|\left(N^{p-2} \frac{d}{d t}\left\langle M_{N}(t)\right\rangle-t p !\right) \mathbb{1}_{\left\{A_{a, b}^{N}\right\}}\right| \\
& \quad=N^{p-2}\left|V_{N}(t)\right| \exp \left\{-2 M_{N}(t)+\left\langle M_{N}(t)\right\rangle\right\} \mathbb{1}_{\left\{A_{a, b}^{N}\right\}} \\
& \leq N^{p-2} \exp \{2 a\}\left|V_{N}(t)\right| \exp \left\{(1+b)\left\langle M_{N}(t)\right\rangle\right\} .
\end{aligned}
$$


Let us also introduce the function $\chi_{b}(x):=[1-\exp \{(1+b) x\}][1+b]^{-1}$. Then, by (2.35) for all $t \leq T$,

$$
\begin{aligned}
& \left|N^{p-2} \chi_{b}\left(\left\langle M_{N}(t)\right\rangle-t^{2} N^{2-p} p ! / 2\right) \mathbb{1}_{\left\{A_{a, b}^{N}\right\}}\right| \\
& =N^{p-2} \mid \int_{0}^{t}\left(\frac{d}{d s}\left\langle M_{N}(s)\right\rangle-s N^{2-p} p !\right) \mathbb{1}_{\left\{A_{a, b}^{N}\right\}} \\
& \quad \times \exp \left\{-(1+b)\left(\left\langle M_{N}(s)\right\rangle-s^{2} N^{2-p} p ! / 2\right)\right\} d s \mid \\
& \leq N^{p-2} \int_{0}^{t}\left|\frac{d}{d s}\left\langle M_{N}(s)\right\rangle-s N^{2-p} p !\right| \mathbb{1}_{\left\{A_{a, b}^{N}\right\}} \\
& \quad \times \exp \left\{-(1+b)\left(\left\langle M_{N}(s)\right\rangle-s^{2} N^{2-p} p ! / 2\right)\right\} d s \\
& \leq N^{(p-2) / 2} \exp \left\{2 a+T N^{(2-p) / 2}(1+b)\right\} \int_{0}^{t}\left|V_{N}(s)\right| d s .
\end{aligned}
$$

This yields

$$
\begin{aligned}
& N^{p-2} \sup _{0 \leq t \leq T}\left|\chi_{b}\left(\left\langle M_{N}(t)\right\rangle-t^{2} N^{2-p} p ! / 2\right)\right| \mathbb{1}_{\left\{A_{a, b}^{N}\right\}} \\
& \quad \leq N^{p-2} \exp \left\{2 a+T N^{(2-p) / 2}(1+b)\right\} \int_{0}^{T}\left|V_{N}(s)\right| d s .
\end{aligned}
$$

We will show in Proposition 2.3 that $\lim _{N \uparrow+\infty} N^{p-2} \mathbb{E}\left|V_{N}(t)\right|=0$ uniformly in $t \in$ $[0, T]$. Consequently, $\sup _{N>1, t \leq T} N^{p-2} \mathbb{E}\left|V_{N}(t)\right|<\infty$. Then, by the dominated convergence theorem,

$$
\lim _{N \uparrow+\infty} \mathbb{E}\left[N^{p-2} \sup _{0 \leq t \leq T}\left|\chi_{b}\left(\left\langle M_{N}(t)\right\rangle-t^{2} N^{2-p} p ! / 2\right)\right| \mathbb{1}_{\left\{A_{a, b}^{N}\right\}}\right]=0 .
$$

It follows that, for all $a, b>0$,

$$
N^{p-2} \sup _{0 \leq t \leq T}\left|\chi_{b}\left(\left\langle M_{N}(t)\right\rangle-t^{2} N^{2-p} p ! / 2\right)\right| \mathbb{1}_{\left\{A_{a, b}^{N}\right\}} \rightarrow 0 \quad \text { as } N \uparrow+\infty
$$

in probability. Then also $N^{p-2} \sup _{0 \leq t \leq T}\left|\chi_{b}\left(\left\langle M_{N}(t)\right\rangle-t^{2} N^{2-p} p ! / 2\right)\right| \rightarrow 0$ as $N \rightarrow \infty$, since by (2.34) the probability of the events $A_{a, b}^{N}$ can be made arbitrarily close to 1 . This last fact implies (2.33) and the lemma is proved.

It remains to prove the following proposition.

Proposition 2.3. Assume that $T$ satisfies (2.32). Then

$$
\lim _{N \uparrow+\infty} N^{p-2} \mathbb{E}\left|V_{N}(t)\right|=0
$$

uniformly in $[0, T]$. 
Proof. It follows from (2.14) and the definition of $V_{N}(t)$ that

$$
N^{p-2} V_{N}(t)=\mathbb{E}_{\sigma, \sigma^{\prime}}\left(N^{p-1} f_{N}^{p}\left(R_{N}\left(\sigma, \sigma^{\prime}\right)\right)-t p !\right) e^{H_{N}(t, \sigma)+H_{N}\left(t, \sigma^{\prime}\right)-N t} .
$$

By the Cauchy-Schwarz inequality,

$$
\begin{array}{rl}
N^{p-2} & \mathbb{E}\left|V_{N}(t)\right| \\
= & \mathbb{E}\left|\mathbb{E}_{\sigma} e^{H_{N}(t, \sigma)-N t / 2} \mathbb{E}_{\sigma^{\prime}}\left(N^{p-1} f_{N}^{p}\left(R_{N}\left(\sigma, \sigma^{\prime}\right)\right)-t p !\right) e^{H_{N}\left(t, \sigma^{\prime}\right)-N t / 2}\right| \\
\leq & {\left[\mathbb{E} \mathbb{E}_{\sigma} e^{H_{N}(t, \sigma)-N t / 2}\right]^{1 / 2}} \\
& \times\left[\mathbb { E } _ { \sigma } e ^ { H _ { N } ( t , \sigma ) - N t / 2 } \left[\mathbb{E}_{\sigma^{\prime}}\left(N^{p-1} f_{N}^{p}\left(R_{N}\left(\sigma, \sigma^{\prime}\right)\right)-t p !\right)\right.\right. \\
& \left.\left.\times e^{H_{N}\left(t, \sigma^{\prime}\right)-N t / 2}\right]^{2}\right]^{1 / 2} \\
= & {\left[\mathbb{E}_{\sigma, \sigma^{\prime}, \sigma^{\prime \prime}}\left(N^{p-1} f_{N}^{p}\left(R_{N}\left(\sigma, \sigma^{\prime}\right)\right)-t p !\right)\left(N^{p-1} f_{N}^{p}\left(R_{N}\left(\sigma, \sigma^{\prime \prime}\right)\right)-t p !\right)\right.} \\
& \left.\times e^{N t\left(f_{N}^{p}\left(R_{N}\left(\sigma, \sigma^{\prime}\right)\right)+f_{N}^{p}\left(R_{N}\left(\sigma, \sigma^{\prime \prime}\right)\right)+f_{N}^{p}\left(R_{N}\left(\sigma^{\prime}, \sigma^{\prime \prime}\right)\right)\right)}\right]^{1 / 2} .
\end{array}
$$

Then it suffices to prove that

$$
\begin{aligned}
W_{N}(t)= & \mathbb{E}_{\sigma, \sigma^{\prime}, \sigma^{\prime \prime}}\left(N^{p-1} f_{N}^{p}\left(R_{N}\left(\sigma, \sigma^{\prime}\right)\right)-t p !\right)\left(N^{p-1} f_{N}^{p}\left(R_{N}\left(\sigma, \sigma^{\prime \prime}\right)\right)-t p !\right) \\
& \times e^{N t\left(f_{N}^{p}\left(R_{N}\left(\sigma, \sigma^{\prime}\right)\right)+f_{N}^{p}\left(R_{N}\left(\sigma, \sigma^{\prime \prime}\right)\right)+f_{N}^{p}\left(R_{N}\left(\sigma^{\prime}, \sigma^{\prime \prime}\right)\right)\right)}
\end{aligned}
$$

tends to 0 uniformly in $[0, T]$ as $N \uparrow+\infty$. We represent it as

$$
\begin{aligned}
W_{N}(t)=\sum_{m_{1}, m_{2}, m_{3} \in \mathcal{A}_{N}} & \left(N^{p-1} f_{N}^{p}\left(m_{1}\right)-t p !\right)\left(N^{p-1} f_{N}^{p}\left(m_{2}\right)-t p !\right) \\
& \times e^{N t\left(f_{N}^{p}\left(m_{1}\right)+f_{N}^{p}\left(m_{2}\right)+f_{N}^{p}\left(m_{3}\right)\right)} \mathbb{P}_{m_{1}, m_{2}, m_{3}},
\end{aligned}
$$

where, by a standard combinatorial calculation,

$$
\begin{aligned}
\mathbb{P}_{m_{1}, m_{2}, m_{3}} \equiv & \mathbb{P}\left\{\sigma \cdot \sigma^{\prime}=m_{1} N, \sigma \cdot \sigma^{\prime \prime}=m_{2} N, \sigma^{\prime} \cdot \sigma^{\prime \prime}=m_{3} N\right\} \\
= & 2^{-2 N}\left(\begin{array}{c}
N \\
N\left(1+m_{1}\right) / 2
\end{array}\right)\left(\begin{array}{c}
N\left(1+m_{1}\right) / 2 \\
N\left(1+m_{1}+m_{2}+m_{3}\right) / 4
\end{array}\right) \\
& \times\left(\begin{array}{c}
N\left(1-m_{1}\right) / 2 \\
N\left(1+m_{2}-m_{1}-m_{3}\right) / 4
\end{array}\right)
\end{aligned}
$$


for $m_{1}, m_{2}, m_{3} \in \mathcal{A}_{N}=\mathcal{A} \cap\{0, \pm 1 / N, \pm 2 / N, \ldots, \pm 1\}^{3}$. By Stirling's formula we obtain

$$
\begin{aligned}
\mathbb{P}_{m_{1}, m_{2}, m_{3}}= & \frac{16 \exp \left\{-N I\left(m_{1}, m_{2}, m_{3}\right)\right\}}{\sqrt{(2 \pi)^{3} N^{3}}} \\
& \times\left[\left(1+m_{1}+m_{2}+m_{3}\right)\left(1-m_{1}-m_{2}+m_{3}\right)\right]^{-1 / 2} \\
& \times\left[\left(1+m_{1}-m_{2}-m_{2}\right)\left(1-m_{1}+m_{2}-m_{3}\right)\right]^{-1 / 2} \\
& \times\left(1+O\left(\frac{1}{N}\right)\right) \quad \text { as } N \uparrow+\infty
\end{aligned}
$$

for any given $m_{1}, m_{2}, m_{3} \in \mathcal{A}_{N}$. Let us note that

$$
I\left(m_{1}, m_{2}, m_{3}\right)-\left(m_{1}^{2}+m_{2}^{2}+m_{3}^{2}\right) / 2=O\left(\left(\left|m_{1}\right|+\left|m_{2}\right|+\left|m_{3}\right|\right)^{3}\right)
$$

as $m_{1}, m_{2}, m_{3} \rightarrow 0$. Then, for all sufficiently small $\varepsilon>0$, there exists a constant $h>0$ such that

$$
\sup _{t \in[0, T]}\left[t\left(m_{1}^{p}+m_{2}^{p}+m_{3}^{p}\right)-I\left(m_{1}, m_{2}, m_{3}\right)\right]<-h\left(m_{1}^{2}+m_{2}^{2}+m_{3}^{2}\right) / 2
$$

for all $m_{1}, m_{2}, m_{3} \in \mathcal{A} \cap\left\{\left|m_{1}\right|+\left|m_{2}\right|+\left|m_{3}\right|<\varepsilon\right\}$. Let us fix such a small $\varepsilon>0$ and an arbitrary constant $0<\delta<1 / 6$ and then split $W_{N}(t)$ into three terms, $W_{N}(t)=I_{N}^{1}(t)+I_{N}^{2}(t)+I_{N}^{3}(t)$, where

$$
\begin{aligned}
& I_{N}^{1}(t)=\sum_{\substack{m_{1}, m_{2}, m_{3} \in \mathcal{A}_{N} \\
\left|m_{1}\right|+\left|m_{2}\right|+\left|m_{3}\right|<N^{-1 / 3-\delta}}}\left(N^{p-1} f_{N}^{p}\left(m_{1}\right)-t p !\right)\left(N^{p-1} f_{N}^{p}\left(m_{2}\right)-t p !\right) \\
& \times e^{t N\left(f_{N}^{p}\left(m_{1}\right)+f_{N}^{p}\left(m_{2}\right)+f_{N}^{p}\left(m_{3}\right)\right)} \mathbb{P}_{m_{1}, m_{2}, m_{3}}, \\
& I_{N}^{2}(t)=\sum_{\substack{m_{1}, m_{2}, m_{3} \in \mathcal{A}_{N} \\
N^{-1 / 3-\delta}<\left|m_{1}\right|+\left|m_{2}\right|+\left|m_{3}\right|<\varepsilon}}\left(N^{p-1} f_{N}^{p}\left(m_{1}\right)-t p !\right)\left(N^{p-1} f_{N}^{p}\left(m_{2}\right)-t p !\right) \\
& \times e^{t N\left(f_{N}^{p}\left(m_{1}\right)+f_{N}^{p}\left(m_{2}\right)+f_{N}^{p}\left(m_{3}\right)\right)} \mathbb{P}_{m_{1}, m_{2}, m_{3}}, \\
& I_{N}^{3}(t)=\sum_{\substack{m_{1}, m_{2}, m_{3} \in \mathcal{A}_{N} \\
\left|m_{1}\right|+\left|m_{2}\right|+\left|m_{3}\right|>\varepsilon}}\left(N^{p-1} f_{N}^{p}\left(m_{1}\right)-t p !\right)\left(N^{p-1} f_{N}^{p}\left(m_{2}\right)-t p !\right) \\
& \times e^{t N\left(f_{N}^{p}\left(m_{1}\right)+f_{N}^{p}\left(m_{2}\right)+f_{N}^{p}\left(m_{3}\right)\right)} \mathbb{P}_{m_{1}, m_{2}, m_{3}} .
\end{aligned}
$$

We will show that $I_{N}^{1}(t), I_{N}^{2}(t)$ and $I_{N}^{3}(t)$ converge to 0 uniformly in $[0, T]$ as $N \rightarrow \infty$.

To prove this for the first term, let us replace the exponent $\exp \left(t N\left(f_{N}^{p}\left(m_{1}\right)+\right.\right.$ $\left.\left.f_{N}^{p}\left(m_{2}\right)+f_{N}^{p}\left(m_{3}\right)\right)\right)$ by its Taylor expansion. We then multiply all expansion terms up to the second order by $\left(N^{p-1} f_{N}^{p}\left(m_{1}\right)-t p !\right)\left(N^{p-1} f_{N}^{p}\left(m_{2}\right)-t p !\right)$ and open the brackets getting 40 terms. Let us show that all but four of them are at most $O(1 / N)$. First, all terms that can be written as $\sum_{\left|m_{1}\right|+\left|m_{2}\right|+\left|m_{3}\right|<N^{-1 / 3-\delta}}\left(f_{N}^{p}\left(m_{i}\right)\right) \times$ 
$\left(f_{N}^{p}\left(m_{j}\right)\right)^{n} \mathbb{P}_{m_{1}, m_{2}, m_{3}}$, with $i, j=1,2,3, i \neq j, n=0,1,2,3$, are exponentially small. In fact, by (2.10) and the independence of the random variables $\sigma \cdot \sigma^{\prime}, \sigma \cdot \sigma^{\prime \prime}$ and $\sigma^{\prime} \cdot \sigma^{\prime \prime}$ in pairs on $\Sigma_{N}^{3}$ under the uniform product measure,

$$
\begin{aligned}
& \quad \sum_{m_{1}, m_{2}, m_{3} \in \mathcal{A}_{N}}\left(f_{N}^{p}\left(m_{i}\right)\right)\left(f_{N}^{p}\left(m_{j}\right)\right)^{n} \mathbb{P}_{m_{1}, m_{2}, m_{3}} \\
& =\mathbb{E}_{\sigma, \sigma^{\prime}, \sigma^{\prime \prime}} f_{N}^{p}\left(R_{N}\left(\sigma, \sigma^{\prime}\right)\right) \mathbb{E}_{\sigma, \sigma^{\prime}, \sigma^{\prime \prime}}\left(f_{N}^{p}\left(R_{N}\left(\sigma, \sigma^{\prime \prime}\right)\right)\right)^{n}=0 .
\end{aligned}
$$

Moreover, by (2.40) and (2.41),

$$
\begin{aligned}
& \mathbb{P}\left(\left|R_{N}\left(\sigma, \sigma^{\prime}\right)\right|+\left|R_{N}\left(\sigma, \sigma^{\prime \prime}\right)\right|+\left|R_{N}\left(\sigma^{\prime}, \sigma^{\prime \prime}\right)\right|>N^{-1 / 3-\delta}\right) \\
& \quad \leq \exp \left\{-h^{\prime} N^{1 / 3-2 \delta}\right\}
\end{aligned}
$$

for some $h^{\prime}>0$. To consider other obtained terms, we note that $\mathbb{E}_{\sigma} \sigma_{i_{1}} \cdots \sigma_{i_{p}}$ equals 1 if all indices of the set $i_{1}, \ldots, i_{p}$ have pairs and 0 otherwise. Then

$$
\begin{aligned}
& \mathbb{E}_{\sigma, \sigma^{\prime}, \sigma^{\prime \prime}}\left(f_{N}^{p}\left(R_{N}\left(\sigma, \sigma^{\prime}\right)\right)\right)^{3}\left(\begin{array}{c}
N \\
p
\end{array}\right)^{-3} \mathbb{E}_{\sigma, \sigma^{\prime}, \sigma^{\prime \prime}}\left(\sum_{i_{1}<i_{2}<\cdots<i_{p}} \sigma_{i_{1}} \sigma_{i_{1}}^{\prime} \cdots \sigma_{i_{p}} \sigma_{i_{p}}^{\prime}\right)^{3} \\
&= C(p)\left(\begin{array}{c}
N \\
p
\end{array}\right)^{-3}\left(\begin{array}{c}
N \\
p
\end{array}\right)\left(\begin{array}{c}
N \\
p / 2
\end{array}\right)=O\left(N^{-3 p / 2}\right), \\
& \mathbb{E}_{\sigma, \sigma^{\prime}, \sigma^{\prime \prime}} f_{N}^{p}\left(R_{N}\left(\sigma, \sigma^{\prime}\right)\right) f_{N}^{p}\left(R_{N}\left(\sigma, \sigma^{\prime \prime}\right)\right) f_{N}^{p}\left(R_{N}\left(\sigma^{\prime}, \sigma^{\prime \prime}\right)\right) \\
&=\left(\begin{array}{c}
N \\
p
\end{array}\right)^{-3}\left(\begin{array}{c}
N \\
p
\end{array}\right)=O\left(N^{-2 p}\right), \\
& \mathbb{E}_{\sigma, \sigma^{\prime}, \sigma^{\prime \prime}} f_{N}^{p}\left(R_{N}\left(\sigma, \sigma^{\prime}\right)\right) f_{N}^{p}\left(R_{N}\left(\sigma, \sigma^{\prime \prime}\right)\right)\left(f_{N}^{p}\left(R_{N}\left(\sigma^{\prime}, \sigma^{\prime \prime}\right)\right)\right)^{2} \\
&=C^{\prime}(p)\left(\begin{array}{c}
N \\
p
\end{array}\right)^{-4}\left(\begin{array}{c}
N \\
p
\end{array}\right)\left(\begin{array}{c}
N \\
p / 2
\end{array}\right)=O\left(N^{-5 p / 2}\right),
\end{aligned}
$$

with some $C(p)>0, C^{\prime}(p)>0$ for $p$ even and $C(p)=C^{\prime}(p)=0$ for $p$ odd. Thus, again by the argument (2.43),

$$
\begin{aligned}
& \sum_{\left|m_{1}\right|+\left|m_{2}\right|+\left|m_{3}\right|<N^{-1 / 3-\delta}} N^{p+1}\left(f_{N}^{p}\left(m_{1}\right)\right)^{3} \mathbb{P}_{m_{1}, m_{2}, m_{3}} \\
& =O\left(N^{1-p / 2}\right), \\
& \sum_{\left|m_{1}\right|+\left|m_{2}\right|+\left|m_{3}\right|<N^{-1 / 3-\delta}} N^{2 p-1} f_{N}^{p}\left(m_{1}\right) f_{N}^{p}\left(m_{2}\right) f_{N}^{p}\left(m_{3}\right) \mathbb{P}_{m_{1}, m_{2}, m_{3}} \\
& =O\left(N^{(1-p) / 2}\right),
\end{aligned}
$$




$$
\begin{aligned}
& \quad \sum_{\left|m_{1}\right|+\left|m_{2}\right|+\left|m_{3}\right|<N^{-1 / 3-\delta}} N^{2 p} f_{N}^{p}\left(m_{1}\right) f_{N}^{p}\left(m_{2}\right)\left(f_{N}^{p}\left(m_{3}\right)\right)^{2} \mathbb{P}_{m_{1}, m_{2}, m_{3}} \\
& =O\left(N^{-p / 2}\right), \\
& \sum_{\left|m_{1}\right|+\left|m_{2}\right|+\left|m_{3}\right|<N^{-1 / 3-\delta}} N^{2}\left(f_{N}^{p}\left(m_{1}\right)\right)^{2} \mathbb{P}_{m_{1}, m_{2}, m_{3}} \\
& =O\left(N^{2-p}\right) .
\end{aligned}
$$

These observations let survive only four terms and lead to the following representation:

$$
\begin{aligned}
I_{N}^{1}(t)= & \sum_{\left|m_{1}\right|+\left|m_{2}\right|+\left|m_{3}\right|<N^{-1 / 3-\delta}}\left(t^{2} N^{2 p}\left(f_{N}^{p}\left(m_{1}\right) f_{N}^{p}\left(m_{2}\right)\right)^{2}-t^{2} N^{p}\left(f_{N}^{p}\left(m_{1}\right)\right)^{2} p !\right. \\
& \left.-t^{2} N^{p}\left(f_{N}^{p}\left(m_{2}\right)\right)^{2} p !+t^{2}(p !)^{2}\right) \mathbb{P}_{m_{1}, m_{2}, m_{3}} \\
& +\sum_{\substack{\left|m_{1}\right|+\left|m_{2}\right|+\left|m_{3}\right|<N^{-1 / 3-\delta}\\
}}\left(N^{p-1} f_{N}^{p}\left(m_{1}\right)-t p !\right)\left(N^{p-1} f_{N}^{p}\left(m_{2}\right)-t p !\right) \\
& \times O\left(t N\left(f_{N}^{p}\left(m_{1}\right)+f_{N}^{p}\left(m_{2}\right)+f_{N}^{p}\left(m_{3}\right)\right)^{3}\right) \mathbb{P}_{m_{1}, m_{2}, m_{3}}+O\left(N^{-1}\right) .
\end{aligned}
$$

By the property of the covariances (2.11) and the independence of $\sigma \cdot \sigma^{\prime}$ and $\sigma \cdot \sigma^{\prime \prime}$, the first term in (2.46) equals $\left(t^{2} N^{2 p}\left(\begin{array}{l}N \\ p\end{array}\right)^{-2}-t^{2} p ! N^{p}\left(\begin{array}{c}N \\ p\end{array}\right)^{-1}-t^{2} p ! N^{p}\left(\begin{array}{c}N \\ p\end{array}\right)^{-1}+\right.$ $\left.t^{2}(p !)^{2}\right)$, up to exponentially small terms, as again the argument (2.43) applies. Therefore this term in (2.46) vanishes.

To investigate the second term in (2.46), one has $t N f_{N}^{p}(m)=o(1)$ for $m=O\left(N^{-1 / 3-\delta}\right), p \geq 3$ and also $N^{p / 2} f_{N}^{p}(m)=\sum_{k=0}^{[p / 2]} d_{p-2 k}(m \sqrt{N})^{p-2 k}(1+$ $O(1 / N))$ by Proposition 2.1. Let us put $m_{i} \sqrt{N}=s_{i}, i=1,2$, 3. Since, by (2.40) and (2.41),

$$
\sup _{\left|m_{1}\right|+\left|m_{2}\right|+\left|m_{3}\right|<N^{-1 / 3-\delta}}\left|\mathbb{P}_{m_{1}, m_{2}, m_{3}} e^{N\left(m_{1}^{2}+m_{2}^{2}+m_{3}^{2}\right) / 2}(2 \pi N)^{3 / 2} / 16-1\right|=O\left(N^{-3 \delta}\right),
$$

this term can be estimated up to some constant and terms of smaller order by

$$
\begin{aligned}
\frac{N^{1-p / 2}}{\sqrt{(2 \pi N)^{3}}} \sum_{\substack{s_{1}, s_{2}, s_{3}=0, \pm 1 / \sqrt{N}, \pm 2 / \sqrt{N}, \ldots \\
\left|s_{1}\right|+\left|s_{2}\right|+\left|s_{3}\right|<N^{1 / 6-\delta}}}\left(\left|t^{3} D_{p}\left(s_{1}\right) D_{p}\left(s_{2}\right)\right|+N^{1-p / 2}\left(\left|t^{4} p ! D_{p}\left(s_{1}\right)\right|\right.\right. \\
\\
\times\left(\left|D_{p}\left(s_{1}\right)\right|+\left|D_{p}\left(s_{2}\right)\right|+\left|D_{p}\left(s_{3}\right)\right|\right)^{3} e^{-\left(s_{1}^{2}+s_{2}^{2}+s_{3}^{2}\right) / 2}\left(1+O\left(N^{-3 \delta}\right)\right),
\end{aligned}
$$

where the polynomial $D_{p}\left(s_{i}\right) \equiv \sum_{k=0}^{[p / 2]} d_{p-2 k} s_{i}^{p-2 k}, i=1,2,3$. The last sum is the integral sum over the density of three independent Gaussian random variables and it converges to the corresponding finite integral. Thus the second term in (2.46) is $O\left(N^{1-p / 2}\right)$ uniformly in $t \in[0, T]$. The analysis of $I_{1}^{N}(t)$ is finished. 
Next, let us study $I_{N}^{2}(t)$. Due to (2.40), the expansion (2.9) and the choice of $\varepsilon>0$ according to (2.42), there exist constants $C_{2}, h_{2}>0$ such that, for all sufficiently large $N$,

$$
\begin{aligned}
& \sup _{\substack{t \in[0, T] \\
m_{1}|+| m_{2}|+| m_{3}\left|>N^{-1 / 3-\delta}\\
\right| m_{1}|+| m_{2}|+| m_{3} \mid<\varepsilon}} \exp \left\{N t\left(f_{N}^{p}\left(m_{1}\right)+f_{N}^{p}\left(m_{2}\right)+f_{N}^{p}\left(m_{3}\right)\right)\right\} \mathbb{P}_{m_{1}, m_{2}, m_{3}} \\
& \leq C_{2} \sup _{\substack{\left|m_{1}\right|+\left|m_{2}\right|+\left|m_{3}\right|>N^{-1 / 3-\delta} \\
\left|m_{1}\right|+\left|m_{2}\right|+\left|m_{3}\right|<\varepsilon}} \exp \left\{-N h\left(m_{1}^{2}+m_{2}^{2}+m_{3}^{2}\right) / 2\right\} \\
& \leq C_{2} \exp \left\{-h_{2} N^{1 / 3-2 \delta}\right\} .
\end{aligned}
$$

The remaining sum in this term has polynomial growth as $N \uparrow+\infty$, and the uniform convergence $I_{N}^{2}(t) \rightarrow 0$ in $[0, T]$ is proved.

Finally, let us consider $I_{N}^{3}(t)$. By Stirling's formula, $\mathbb{P}_{m_{1}, m_{2}, m_{3}} \leq C \sqrt{N}$ $\times e^{-N I\left(m_{1}, m_{2}, m_{3}\right)}$ for some $C>0$ and all $\left(m_{1}, m_{2}, m_{3}\right) \in \mathcal{A}_{N} \cap\left\{\left|m_{1}\right|+\left|m_{2}\right|+\right.$ $\left.\left|m_{3}\right|>\varepsilon\right\}$. Then, by (2.9) and the assumption (2.32), for given $T$ there exist constants $C_{3}>0, h_{3}>0$ such that

$$
\begin{aligned}
& \sup _{t \in[0, T],\left|m_{1}\right|+\left|m_{2}\right|+\left|m_{3}\right|>\varepsilon} e^{N t\left(f_{N}^{p}\left(m_{1}\right)+f_{N}^{p}\left(m_{2}\right)+f_{N}^{p}\left(m_{3}\right)\right)} \mathbb{P}_{m_{1}, m_{2}, m_{3}} \\
& \leq C_{3} \sqrt{N} \sup _{t \in[0, T],\left|m_{1}\right|+\left|m_{2}\right|+\left|m_{3}\right|>\varepsilon} e^{N\left[t\left(m_{1}^{p}+m_{2}^{p}+m_{3}^{p}\right)-I\left(m_{1}, m_{2}, m_{3}\right)\right]} \\
& <C_{3} \sqrt{N} e^{-h_{3} N} \text {. }
\end{aligned}
$$

The remaining sum in this term has again polynomial growth, whence $I_{N}^{3}(t) \rightarrow 0$ uniformly in $[0, T]$. The proposition is proved.

REMARK. Let us note that the restriction (2.32) on $T$ was essential only for the analysis of the third term $I_{N}^{3}(t)$. This means that the convergence $N^{p-2} \mathbb{E}\left|V_{N}(t)\right| \rightarrow 0$ breaks down for larger $T$ only because of the configurations of spins with rather big overlaps $\sigma \cdot \sigma^{\prime}, \sigma \cdot \sigma^{\prime \prime}, \sigma^{\prime} \cdot \sigma^{\prime \prime}$. To extend our result to the whole interval (2.20) of admissible $T$, we need to reduce the contribution of these configurations into $W_{N}(t)$. For that purpose we will follow Talagrand's [27] idea of truncating the Hamiltonian.

Now we prove the statement of the previous lemma for all $T$ satisfying (2.20).

LEMMA 2.4. Let

$$
T<\inf _{m_{1}, m_{2}, m_{3} \in \mathcal{A}} Y_{p}\left(m_{1}, m_{2}, m_{3}\right) .
$$


Then

$$
\sup _{0 \leq t \leq T}\left|\left\langle N^{(p-2) / 2} M_{N}(t)\right\rangle-t^{2} p ! / 2\right| \rightarrow 0
$$

in probability.

PROOF. Let us fix $\varepsilon>0$ such that, for some constants $h_{1}, h_{2}>0$,

$$
\begin{aligned}
& \sup _{t \in[0, T], m_{1}^{p}+m_{2}^{p}+m_{3}^{p}<3 \varepsilon}\left[t\left(m_{1}^{p}+m_{2}^{p}+m_{3}^{p}\right)-I\left(m_{1}, m_{2}, m_{3}\right)\right] \\
& <-h_{1}\left(m_{1}^{2}+m_{2}^{2}+m_{3}^{2}\right)
\end{aligned}
$$

and

$$
\begin{aligned}
& \sup _{\substack{t \in[0, T], m_{1}, m_{2}, m_{3} \in \mathcal{A} \\
m_{1}^{p}+m_{2}^{p}+m_{3}^{p}>3 \varepsilon}} t\left[\operatorname { m i n } \left\{Q\left(m_{1}^{p}, m_{2}^{p}, m_{3}^{p}, \varepsilon\right), L\left(m_{1}^{p}, m_{2}^{p}, m_{3}^{p}, \varepsilon\right),\right.\right. \\
& \left.\left.\quad L\left(m_{1}^{p}, m_{3}^{p}, m_{2}^{p}, \varepsilon\right), L\left(m_{2}^{p}, m_{3}^{p}, m_{1}^{p}, \varepsilon\right)\right\}\right] \\
& -I\left(m_{1}, m_{2}, m_{3}\right)<-h_{2},
\end{aligned}
$$

where

$$
\begin{aligned}
Q\left(m_{1}, m_{2}, m_{3}, \varepsilon\right)= & {\left[-9 \varepsilon^{2}+6(1+2 \varepsilon)\left(m_{1}+m_{2}+m_{3}\right)\right] } \\
& \times\left[2\left(3+2 m_{1}+2 m_{2}+2 m_{3}\right)\right]^{-1}, \\
L\left(m_{1}, m_{2}, m_{3}, \varepsilon\right)= & {\left[-1-m_{3}-(1+\varepsilon)^{2}+(1+\varepsilon) S\left(m_{1}, m_{2}, m_{3}\right) \sqrt{2+2 m_{3}}\right.} \\
& \left.+R\left(m_{1}, m_{2}, m_{3}\right)\left(1+m_{3}\right)\right]\left[1+m_{3}\right]^{-1} .
\end{aligned}
$$

Condition (2.48) is the same as (2.42) and, due to (2.41), for any given $T>0$ it is possible to find an appropriate $\varepsilon>0$ such that (2.48) is satisfied. However, $\varepsilon>0$ ensuring (2.49) exists, if and only if $T$ satisfies the assumption (2.20). The meaning of (2.49) will become clear in the proof of Proposition 2.5. Let us introduce

$$
\begin{aligned}
\widetilde{V}_{N}(t, \varepsilon)= & \mathbb{E}_{\sigma, \sigma^{\prime}}\left(N f_{N}^{p}\left(R_{N}\left(\sigma, \sigma^{\prime}\right)\right)-N^{2-p} t p !\right) e^{H_{N}(t, \sigma)+H_{N}\left(t, \sigma^{\prime}\right)-N t} \\
& \times \mathbb{1}_{\left\{H_{N}(t, \sigma)<(1+\varepsilon) t N, H_{N}\left(t, \sigma^{\prime}\right)<(1+\varepsilon) t N\right\}}, \\
\bar{V}_{N}(t, \varepsilon)= & \mathbb{E}_{\sigma, \sigma^{\prime}}\left(N f_{N}^{p}\left(R_{N}\left(\sigma, \sigma^{\prime}\right)\right)-N^{2-p} t p !\right) e^{H_{N}(t, \sigma)+H_{N}\left(t, \sigma^{\prime}\right)-N t} \\
& \times \mathbb{1}_{\left\{H_{N}(t, \sigma)>(1+\varepsilon) t N \text { or } H_{N}\left(t, \sigma^{\prime}\right)>(1+\varepsilon) t N\right\}} \\
= & V_{N}(t)-\widetilde{V}_{N}(t, \varepsilon) .
\end{aligned}
$$

Let us also fix some $T_{0}>0$ satisfying assumption (2.32) of the previous lemma. Proceeding along the lines of the proof of Lemma 2.2, we get, for all $t \in\left[T_{0}, T\right]$, 


$$
\begin{aligned}
& N^{p-2}\left|\chi_{b}\left(\left\langle M_{N}(t)\right\rangle-t^{2} N^{2-p} p ! / 2\right)\right| \mathbb{1}_{\left\{A_{a, b}^{N}\right\}} \\
& \leq N^{p-2} \exp \left\{2 a+T_{0} N^{2-p}(1+b)\right\} \\
& \quad \times \int_{0}^{T_{0}}\left|V_{N}(s)\right| d s+N^{p-2} \exp \left\{2 a+t N^{2-p}(1+b)\right\} \int_{T_{0}}^{t}\left|\widetilde{V}_{N}(s, \varepsilon)\right| d s \\
& \quad+N^{p-2} \int_{T_{0}}^{t}\left|\bar{V}_{N}(s, \varepsilon)\right| \bar{Z}_{N}^{-2}(s) \\
& \quad \quad \times \exp \left\{-(1+b)\left(\left\langle M_{N}(s)\right\rangle-s^{2} N^{2-p} p ! / 2\right)\right\} \mathbb{1}_{\left\{A_{a, \varepsilon}^{N}\right\}} d s .
\end{aligned}
$$

Then

$$
\begin{aligned}
N^{p-2} & \sup _{T_{0}<t \leq T}\left|\chi_{b}\left(\left\langle M_{N}(t)\right\rangle-t^{2} N^{2-p} p ! / 2\right)\right| \mathbb{1}_{\left\{A_{a, b}^{N}\right\}} \\
\leq & N^{p-2} \exp \left\{2 a+T_{0} N^{2-p}(1+b)\right\} \\
& \times \int_{0}^{T_{0}}\left|V_{N}(s)\right| d s+N^{p-2} \exp \left\{2 a+T N^{2-p}(1+b)\right\} \int_{T_{0}}^{T}\left|\widetilde{V}_{N}(s, \varepsilon)\right| d s \\
& +N^{p-2} \exp \left\{T N^{2-p}(1+b)\right\} \int_{T_{0}}^{T}\left|\bar{V}_{N}(s, \varepsilon)\right| \bar{Z}_{N}^{-2}(s) d s .
\end{aligned}
$$

It was proved in Lemma 2.2 that $N^{p-2} \mathbb{E}\left|V_{N}(t)\right| \rightarrow 0$ uniformly in $\left[0, T_{0}\right]$ as $N \uparrow+\infty$. Proposition 2.5 shows that, for $\varepsilon>0$ satisfying (2.48) and (2.49), $N^{p-2} \mathbb{E}|\widetilde{V}(t, \varepsilon)| \rightarrow 0$ uniformly in $t \in\left[T_{0}, T\right]$. Proposition 2.6 proves that $N^{p-2} \mathbb{E}\left|\bar{V}(t, \varepsilon) Z_{N}^{-2}(t)\right| \rightarrow 0$ uniformly in $\left[T_{0}, T\right]$ for all $\varepsilon>0$. Then

$$
\lim _{N \uparrow+\infty} \mathbb{E}\left[\sup _{T_{0} \leq t \leq T}\left|N^{p-2} \chi_{b}\left(\left\langle M_{N}(t)\right\rangle-t^{2} N^{(2-p) / 2} p ! / 2\right)\right| \mathbb{1}_{\left\{A_{a, b}^{N}\right\}}\right]=0 .
$$

Then $\sup _{0 \leq t \leq T}\left|N^{p-2} \chi_{b}\left(\left\langle M_{N}(t)\right\rangle-t^{2} N^{2-p} p ! / 2\right)\right|$ converges to 0 in probability, since the probability of the events $A_{a, b}^{N}$ can be made arbitrarily close to 1 by (2.34). This implies (2.47) and the proof of the lemma is complete.

Proposition 2.5. Assume that $T>0$ satisfies (2.20). Let us fix $0<\varepsilon<1 / 2$ such that (2.48) and (2.49) hold. Then, for any $T_{0}>0, T_{0}<T$,

$$
\lim _{N \uparrow+\infty} N^{p-2} \mathbb{E}\left|\widetilde{V}_{N}(t, \varepsilon)\right|=0
$$

uniformly in $t \in\left[T_{0}, T\right]$.

Proof. Let us estimate $N^{p-2} \mathbb{E}\left|\widetilde{V}_{N}(t, \varepsilon)\right|$ by the Cauchy-Schwarz inequality as in the proof of Proposition 2.3 for $N^{p-2} \mathbb{E}\left|V_{N}(t)\right|$. After that we split it into four 
terms:

$$
N^{p-2} \mathbb{E}\left|\widetilde{V}_{N}(t, \varepsilon)\right| \leq\left[\widetilde{I}_{N}^{1}(t, \varepsilon)-\widetilde{I}_{N}^{2}(t, \varepsilon)+\widetilde{I}_{N}^{3}(t, \varepsilon)+\widetilde{I}_{N}^{4}(t, \varepsilon)\right]^{1 / 2},
$$

$$
\begin{aligned}
& \tilde{I}_{N}^{1}(t, \varepsilon)=\sum_{\substack{m_{1}, m_{2}, m_{3} \in \mathcal{A}_{N} \\
m_{1}^{p}+m_{2}^{p}+m_{3}^{p} \leq \varepsilon^{2} / 4}}\left(N^{p-1} f_{N}^{p}\left(m_{1}\right)-t p !\right)\left(N^{p-1} f_{N}^{p}\left(m_{2}\right)-t p !\right) \\
& \times \mathbb{P}_{m_{1}, m_{2}, m_{3}} \mathbb{E} e^{H_{N}(t, \sigma)+H_{N}\left(t, \sigma^{\prime}\right)+H_{N}\left(t, \sigma^{\prime \prime}\right)-3 t N / 2}, \\
& \widetilde{I}_{N}^{2}(t, \varepsilon)=\sum_{\substack{m_{1}, m_{2}, m_{3} \in \mathcal{A}_{N} \\
m_{1}^{p}+m_{2}^{p}+m_{3}^{p}<\varepsilon^{2} / 4}}\left(N^{p-1} f_{N}^{p}\left(m_{1}\right)-t p !\right)\left(N^{p-1} f_{N}^{p}\left(m_{2}\right)-t p !\right) \\
& \times \mathbb{P}_{m_{1}, m_{2}, m_{3}} \mathbb{E}\left[e^{H_{N}(t, \sigma)+H_{N}\left(t, \sigma^{\prime}\right)+H_{N}\left(t, \sigma^{\prime \prime}\right)-3 N t / 2}\right.
\end{aligned}
$$

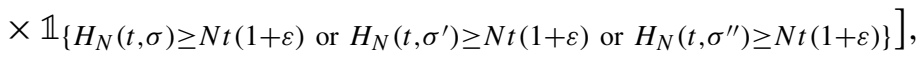

$$
\begin{aligned}
& \tilde{I}_{N}^{3}(t, \varepsilon)=\sum_{\substack{m_{1}, m_{2}, m_{3} \in \mathcal{A}_{N} \\
\varepsilon^{2} / 4 \leq m_{1}^{p}+m_{2}^{p}+m_{3}^{p} \leq 3 \varepsilon}}\left(N^{p-1} f_{N}^{p}\left(m_{1}\right)-t p !\right)\left(N^{N-1} f_{N}^{p}\left(m_{2}\right)-t p !\right) \\
& \times \mathbb{P}_{m_{1}, m_{2}, m_{3}} \mathbb{E}\left[e^{H_{N}(t, \sigma)+H_{N}\left(t, \sigma^{\prime}\right)+H_{N}\left(t, \sigma^{\prime \prime}\right)-3 N t / 2}\right. \\
& \left.\times \mathbb{1}_{\left\{H_{N}(t, \sigma)<N t(1+\varepsilon), H_{N}\left(t, \sigma^{\prime}\right)<N t(1+\varepsilon), H_{N}\left(t, \sigma^{\prime \prime}\right)<N t(1+\varepsilon)\right\}}\right], \\
& \widetilde{I}_{N}^{4}(t, \varepsilon)=\sum_{\substack{m_{1}, m_{2}, m_{3} \in \mathcal{A}_{N} \\
m_{1}^{p}+m_{2}^{p}+m_{3}^{p}>3 \varepsilon}}\left(N^{p-1} f_{N}^{p}\left(m_{1}\right)-t p !\right)\left(N^{p-1} f_{N}^{p}\left(m_{2}\right)-t p !\right) \\
& \times \mathbb{P}_{m_{1}, m_{2}, m_{3}} \mathbb{E}\left[e^{H_{N}(t, \sigma)+H_{N}\left(t, \sigma^{\prime}\right)+H_{N}\left(t, \sigma^{\prime \prime}\right)-3 t N / 2}\right.
\end{aligned}
$$

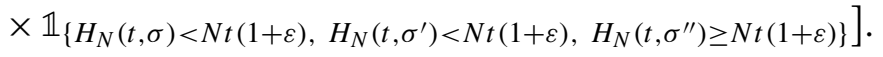

We will prove the uniform convergence to 0 in $\left[T_{0}, T\right]$ as $N \uparrow+\infty$ of all these four terms.

The first term $\widetilde{I}_{N}^{1}(t)$ is not truncated and it refers to the configurations of spins with small correlations $m_{1}^{p}, m_{2}^{p}$ and $m_{3}^{p}$. The proof of its uniform convergence to 0 in $\left[T_{0}, T\right]$ relies on Proposition 2.1, (2.9)-(2.11) and the choice of $\varepsilon$ according to (2.48). It is completely analogous to the proof of the uniform convergence to 0 of the sum $I_{N}^{1}(t)+I_{N}^{2}(t)$ in the proof of Proposition 2.3. Therefore we omit the details.

The second term $\widetilde{I}_{N}^{2}(t)$ also contains only configurations of spins with very small correlations. If these correlations were 0 , that is, if $H_{N}(t, \sigma), H_{N}\left(t, \sigma^{\prime}\right)$ and $H_{N}\left(t, \sigma^{\prime \prime}\right)$ were independent, then, indeed, the expectation involved in this term would satisfy

$$
\begin{aligned}
& \mathbb{E}\left[e^{H_{N}(t, \sigma)+H_{N}\left(t, \sigma^{\prime}\right)+H_{N}\left(t, \sigma^{\prime \prime}\right)-3 N t / 2} \mathbb{1}_{\{\cdot\}}\right] \\
& \leq 3 \mathbb{E}\left[e^{\sqrt{N t \xi}-N t / 2} \mathbb{1}_{\{\xi>\sqrt{N t}(1+\varepsilon)\}}\right] \leq \exp \left\{-N t \varepsilon^{2} / 2\right\}
\end{aligned}
$$


( $\xi$ is a standard Gaussian) by a well-known estimate for Gaussian random variables (B.1). We show that very small correlations $m_{1}, m_{2}, m_{3}$ do not destroy the exponential convergence to 0 of the corresponding expectation. Considering the third term $\widetilde{I}_{N}^{3}(t)$, we neglect the truncation and use the asymptotic expansion (2.40) and condition (2.48). So we prove that the expectation $\mathbb{E} e^{H_{N}(t, \sigma)+H_{N}\left(t, \sigma^{\prime}\right)+H_{N}\left(t, \sigma^{\prime \prime}\right)-3 N t / 2}$ multiplied by the probability of any given correlations goes to 0 exponentially fast. Finally, $\widetilde{I}_{N}^{4}(t)$ refers to the configurations of spins with rather large correlations. Here, applying the estimate (B.1), we benefit from the truncation. The choice of $\varepsilon>0$ according to (2.49) plays a crucial role in the analysis of this term. [Remember that this choice was possible only for $T$ satisfying (2.20).]

Now we proceed with the detailed proof. To treat the second term $\widetilde{I}_{N}^{2}(t, \varepsilon)$, we write

$$
\begin{aligned}
& \mathbb{E}\left[e^{H_{N}(t, \sigma)+H_{N}(t, \sigma)+H_{N}\left(t, \sigma^{\prime \prime}\right)-3 N t / 2}\right. \\
& \left.\times \mathbb{1}_{\left\{H_{N}(t, \sigma)>N t(1+\varepsilon) \text { or } H_{N}\left(t, \sigma^{\prime}\right)>N t(1+\varepsilon) \text { or } H_{N}\left(t, \sigma^{\prime \prime}\right)>N t(1+\varepsilon)\right\}}\right] \\
& \quad=\mathbb{E}\left[e^{\sqrt{N t}\left(\xi_{1}+\xi_{2}+\xi_{3}\right)-3 N t / 2} \mathbb{1}_{\left\{\xi_{1}>\sqrt{N t}(1+\varepsilon) \text { or } \xi_{2}>\sqrt{N t}(1+\varepsilon) \text { or } \xi_{3}>\sqrt{N t}(1+\varepsilon)\right\}}\right],
\end{aligned}
$$

where $\xi_{1}, \xi_{2}$ and $\xi_{3}$ are Gaussian random variables with mean 0 , variance 1 and covariances

$$
\begin{aligned}
& \operatorname{cov}\left(\xi_{1}, \xi_{2}\right)=f_{N}^{p}\left(R_{N}\left(\sigma, \sigma^{\prime}\right)\right)=m_{1}^{p}+O(1 / N), \\
& \operatorname{cov}\left(\xi_{1}, \xi_{3}\right)=f_{N}^{p}\left(R_{N}\left(\sigma, \sigma^{\prime \prime}\right)\right)=m_{2}^{p}+O(1 / N), \\
& \operatorname{cov}\left(\xi_{2}, \xi_{3}\right)=f_{N}^{p}\left(R_{N}\left(\sigma^{\prime}, \sigma^{\prime \prime}\right)\right)=m_{3}^{p}+O(1 / N), \quad m_{1}^{p}+m_{2}^{p}+m_{3}^{p} \leq \varepsilon^{2} / 4 .
\end{aligned}
$$

One gets

$$
\begin{aligned}
& \mathbb{E}\left[e^{\sqrt{N t}\left(\xi_{1}+\xi_{2}+\xi_{3}\right)-3 N t / 2} \mathbb{1}_{\left\{\xi_{1}>\sqrt{N t}(1+\varepsilon)\right\}}\right] \\
& =e^{-3 N t / 2} \mathbb{E}\left[e^{\sqrt{N t} \xi_{1}} \mathbb{1}_{\left\{\xi_{1}>\sqrt{N t}(1+\varepsilon)\right\}} \mathbb{E}\left(e^{\xi_{2}+\xi_{3}} \mid \xi_{1}\right)\right] \\
& =e^{N t \gamma-3 N t / 2} \mathbb{E}\left[e^{\sqrt{N t}(1+\mu) \xi_{1}} \mathbb{1}_{\left\{\xi_{1}>\sqrt{N t}(1+\varepsilon)\right\}}\right],
\end{aligned}
$$

where $\gamma=1+f_{N}^{p}\left(m_{3}\right)-\left(f_{N}^{p}\left(m_{1}\right)+f_{N}^{p}\left(m_{2}\right)\right)^{2} / 2, \mu=f_{N}^{p}\left(m_{1}\right)+f_{N}^{p}\left(m_{2}\right)=$ $m_{1}^{p}+m_{2}^{p}+O(1 / N)$. Since $m_{1}^{p}+m_{2}^{p} \leq \varepsilon^{2} / 4<\varepsilon$, we may use the estimate for standard Gaussian random variables (B.1), which implies

$$
\begin{aligned}
& \mathbb{E}\left[e^{\sqrt{N t}\left(\xi_{1}+\xi_{2}+\xi_{3}\right)-3 N t / 2} \mathbb{1}_{\left\{\xi_{1}>\sqrt{N t}(1+\varepsilon)\right\}}\right] \\
& \quad \leq C_{1} e^{N t\left(f_{N}^{p}\left(m_{1}\right)+f_{N}^{p}\left(m_{2}\right)+f_{N}^{p}\left(m_{3}\right)-\left(\varepsilon-f_{N}^{p}\left(m_{1}\right)+f_{N}^{p}\left(m_{2}\right)\right)^{2} / 2\right)} \\
& \leq C_{1}^{\prime} e^{N t\left(m_{1}^{p}+m_{2}^{p}+m_{3}^{p}-\left(\varepsilon-m_{1}^{p}+m_{2}^{p}\right)^{2} / 2\right)} \leq C_{1}^{\prime} e^{-N T_{0} \varepsilon^{2} / 8}
\end{aligned}
$$


for some constants $C_{1}, C_{1}^{\prime}>0$, all $t \in\left[T_{0}, T\right]$ and all $N>0$, if $m_{1}^{p}+m_{2}^{p}+m_{3}^{p}<$ $\varepsilon^{2} / 4,0<\varepsilon<1 / 2$. Thus

$$
\begin{gathered}
\sup _{0 \leq m_{1}^{p}+m_{2}^{p}+m_{3}^{p} \leq \varepsilon^{2} / 4} \mathbb{E}\left[e^{H_{N}(t, \sigma)+H_{N}(t, \sigma)+H_{N}\left(t, \sigma^{\prime \prime}\right)-3 N t / 2}\right. \\
\left.\quad \times \mathbb{1}_{\left\{H_{N}(t, \sigma)>N t(1+\varepsilon) \text { or } H_{N}\left(t, \sigma^{\prime}\right)>N t(1+\varepsilon) \text { or } H_{N}\left(t, \sigma^{\prime \prime}\right)>N t(1+\varepsilon)\right\}}\right] \\
\leq 3 C_{1}^{\prime} e^{-N T_{0} \varepsilon^{2} / 8}
\end{gathered}
$$

for all $t \in\left[T_{0}, T\right]$. Since the other terms in $\widetilde{I}_{N}^{2}(t, \varepsilon)$ have polynomial growth, the uniform convergence $\widetilde{I}_{N}^{2}(t, \varepsilon) \rightarrow 0$ in $\left[T_{0}, T\right]$ follows.

Let us turn to $\widetilde{I}_{N}^{3}(t, \varepsilon)$. By the expansions (2.40), (2.9) and condition (2.48),

$$
\begin{aligned}
& \sup _{\varepsilon^{2} / 4 \leq m_{1}^{p}+m_{2}^{p}+m_{3}^{p} \leq 3 \varepsilon} \mathbb{E}\left[\exp \left\{H_{N}(t, \sigma)+H_{N}\left(t, \sigma^{\prime}\right)+H_{N}\left(t, \sigma^{\prime \prime}\right)-3 N t / 2\right\}\right. \\
& \quad \times \mathbb{1}_{\left.\left\{H_{N}(t, \sigma)<N t(1+\varepsilon), H_{N}\left(t, \sigma^{\prime}\right)<N t(1+\varepsilon), H_{N}\left(t, \sigma^{\prime \prime}\right)<N t(1+\varepsilon)\right\}\right]} \\
& \times \mathbb{P}_{m_{1}, m_{2}, m_{3}} \\
& \leq C_{2} \sup _{\varepsilon^{2} / 4 \leq m_{1}^{p}+m_{2}^{p}+m_{3}^{p} \leq 3 \varepsilon} \exp \left\{N\left[t\left(f_{N}^{p}\left(m_{1}\right)+f_{N}^{p}\left(m_{2}\right)+f_{N}^{p}\left(m_{3}\right)\right)-I(m)\right]\right\} \\
& \leq C_{2}^{\prime} \sup _{\varepsilon^{2} / 4 \leq m_{1}^{p}+m_{2}^{p}+m_{3}^{p} \leq 3 \varepsilon} \exp \left\{-h_{1} N\left(m_{1}^{2}+m_{2}^{2}+m_{3}^{2}\right)\right\} \\
& \leq C_{2}^{\prime} \exp \left\{-h_{1} \varepsilon^{4 / p} N / 4\right\}
\end{aligned}
$$

for all $t \in\left[T_{0}, T\right]$, where $C_{2}, C_{2}^{\prime}>0$ are constants. All other terms in $\widetilde{I}_{N}^{3}(t, \varepsilon)$ have polynomial growth; hence $I_{N}^{3}(t, \varepsilon) \rightarrow 0$ uniformly in $\left[T_{0}, T\right]$.

Finally, consider $\widetilde{I}_{N}^{4}(t, \varepsilon)$. We have

$$
\begin{aligned}
& \mathbb{E}\left[e^{H_{N}(t, \sigma)+H_{N}\left(t, \sigma^{\prime}\right)+H_{N}\left(t, \sigma^{\prime \prime}\right)-3 N t / 2}\right. \\
& \left.\times \mathbb{1}_{\left\{H_{N}(t, \sigma)<N t(1+\varepsilon), H_{N}\left(t, \sigma^{\prime}\right)<N t(1+\varepsilon), H_{N}\left(t, \sigma^{\prime \prime}\right)<(1+\varepsilon) N t\right\}}\right] \\
& \leq \mathbb{E}\left[e^{\sqrt{N t\left(3+2 f_{N}^{p}\left(m_{1}\right)+2 f_{N}^{p}\left(m_{2}\right)+2 f_{N}^{p}\left(m_{3}\right)\right)} \xi-3 N t / 2}\right. \\
& \left.\quad \times \mathbb{1}_{\left\{\sqrt{3+2 f_{N}^{p}\left(m_{1}\right)+2 f_{N}^{p}\left(m_{2}\right)+2 f_{N}^{p}\left(m_{3}\right)} \xi \leq 3 N t(1+\varepsilon)\right\}}\right] .
\end{aligned}
$$

Since $f_{N}^{p}\left(m_{1}\right)+f_{N}^{p}\left(m_{2}\right)+f_{N}^{p}\left(m_{3}\right)=m_{1}^{p}+m_{2}^{p}+m_{3}^{p}+O(1 / N)>3 \varepsilon$, we may apply the estimate (B.2), which yields

$$
\begin{aligned}
& \mathbb{E}\left[e^{H_{N}(t, \sigma)+H_{N}\left(t, \sigma^{\prime}\right)+H_{N}\left(t, \sigma^{\prime \prime}\right)-3 N t / 2}\right. \\
& \left.\times \mathbb{1}_{\left\{H_{N}(t, \sigma)<N t(1+\varepsilon), H_{N}\left(t, \sigma^{\prime}\right)<N t(1+\varepsilon), H_{N}\left(t, \sigma^{\prime \prime}\right)<(1+\varepsilon) N t\right\}}\right] \\
& \quad \leq C_{3} \exp \left\{N t Q\left(f_{N}^{p}\left(m_{1}\right), f_{N}^{p}\left(m_{2}\right), f_{N}^{p}\left(m_{3}\right), \varepsilon\right)\right\}
\end{aligned}
$$


for some constant $C_{3}>0$, all $t \in\left[T_{0}, T\right], N>0$ and $m_{1}^{p}+m_{2}^{p}+m_{3}^{p}>3 \varepsilon$. On the other hand, we also have

$$
\begin{aligned}
& \mathbb{E}\left[e^{H_{N}(t, \sigma)+H_{N}\left(t, \sigma^{\prime}\right)+H_{N}\left(t, \sigma^{\prime \prime}\right)-3 N t / 2}\right. \\
& \times \mathbb{1}_{\left.\left\{H_{N}(t, \sigma)<N t(1+\varepsilon), H_{N}\left(t, \sigma^{\prime}\right)<N t(1+\varepsilon), H_{N}\left(t, \sigma^{\prime \prime}\right)<(1+\varepsilon) N t\right\}\right]} \\
& \leq \mathbb{E}\left[e^{H_{N}(t, \sigma)+H_{N}\left(t, \sigma^{\prime}\right)+H_{N}\left(t, \sigma^{\prime \prime}\right)-3 N t / 2}\right. \\
& \quad \times \mathbb{1}_{\left.\left\{H_{N}\left(t, \sigma^{\prime}\right)<N t(1+\varepsilon), H_{N}\left(t, \sigma^{\prime \prime}\right)<N t(1+\varepsilon)\right\}\right]} \\
&= \mathbb{E}\left[e^{\sqrt{N t} \xi_{2}+\sqrt{N t} \xi_{3}-3 N t / 2} \mathbb{E}\left(e^{\sqrt{N t} \xi_{1}} \mid \xi_{2}, \xi_{3}\right) \mathbb{1}_{\left.\left\{\xi_{2}<\sqrt{N t}(1+\varepsilon), \xi_{3}<\sqrt{N t}(1+\varepsilon)\right\}\right]}\right. \\
&= e^{-N t+N t \alpha} \mathbb{E}\left[e^{\sqrt{N t}\left(1+\mu_{2}\right) \xi_{2}+\sqrt{N t}\left(1+\mu_{3}\right) \xi_{3}} \mathbb{1}_{\left\{\xi_{2}<\sqrt{N t}(1+\varepsilon), \xi_{3}<\sqrt{N t}(1+\varepsilon)\right\}}\right] \\
& \leq e^{-N t+N t \alpha} \mathbb{E}\left[e^{\sqrt{N t\left(\left(1+\mu_{2}\right)^{2}+\left(1+\mu_{3}\right)^{2}+2 f_{N}^{p}\left(m_{3}\right)\left(1+\mu_{2}\right)\left(1+\mu_{3}\right)\right) \xi}}\right. \\
&\left.\times \mathbb{1}_{\left\{\sqrt{2+2 f_{N}^{p}\left(m_{3}\right)} \xi<2 \sqrt{N t}(1+\varepsilon)\right\}}\right]
\end{aligned}
$$

where $\xi_{1}, \xi_{2}, \xi_{3}$ are the same as in the analysis of the second term, $\xi$ is a standard Gaussian and

$$
\begin{aligned}
\alpha & =\left(2 f_{N}^{p}\left(m_{1}\right) f_{N}^{p}\left(m_{2}\right) f_{N}^{p}\left(m_{3}\right)-\left(f_{N}^{p}\left(m_{1}\right)\right)^{2}-\left(f_{N}^{p}\left(m_{2}\right)\right)^{2}\right) /\left(2-2\left(f_{N}^{p}\left(m_{3}\right)^{2}\right),\right. \\
\mu_{2} & =\left(f_{N}^{p}\left(m_{1}\right)-f_{N}^{p}\left(m_{2}\right) f_{N}^{p}\left(m_{3}\right)\right) /\left(1-\left(f_{N}^{p}\left(m_{3}\right)\right)^{2}\right), \\
\mu_{3} & =\left(f_{N}^{p}\left(m_{2}\right)-f_{N}^{p}\left(m_{1}\right) f_{N}^{p}\left(m_{3}\right)\right) /\left(1-\left(f_{N}^{p}\left(m_{3}\right)^{2}\right) .\right.
\end{aligned}
$$

One checks that

$$
\begin{aligned}
& \sqrt{\left(1+\mu_{2}\right)^{2}+\left(1+\mu_{3}\right)^{2}+2 f_{N}^{p}\left(m_{3}\right)\left(1+\mu_{2}\right)\left(1+\mu_{3}\right)} \\
& \quad \geq \frac{2\left(1+f_{N}^{p}\left(m_{3}\right)+\left(f_{N}^{p}\left(m_{1}\right)+f_{N}^{p}\left(m_{2}\right)\right) / 2\right)}{\sqrt{2+2 f_{N}^{p}\left(m_{3}\right)}} \geq \frac{2(1+3 \varepsilon / 2)}{\sqrt{2+2 f_{N}^{p}\left(m_{3}\right)}}+O\left(\frac{1}{N}\right),
\end{aligned}
$$

when $m_{1}^{p}+m_{2}^{p}+m_{3}^{p}>3 \varepsilon$. So, we are again in the position to apply (B.2). This yields

$$
\begin{aligned}
& \mathbb{E}\left[e^{H_{N}(t, \sigma)+H_{N}\left(t, \sigma^{\prime}\right)+H_{N}\left(t, \sigma^{\prime \prime}\right)-3 N t / 2}\right. \\
& \left.\quad \times \mathbb{1}_{\left\{H_{N}(t, \sigma)<N t(1+\varepsilon), H_{N}\left(t, \sigma^{\prime}\right)<N t(1+\varepsilon), H_{N}\left(t, \sigma^{\prime \prime}\right)<(1+\varepsilon) N t\right\}}\right] \\
& \quad \leq C_{4} e^{t N L\left(f_{N}^{p}\left(m_{1}\right), f_{N}^{p}\left(m_{2}\right), f_{N}^{p}\left(m_{3}\right), \varepsilon\right)},
\end{aligned}
$$

where $C_{4}>0$ is a constant. Permuting $m_{1}, m_{2}$ and $m_{3}$, we can derive in the same way that the same expectation does not exceed $\exp \left\{t N L_{p}\left(f_{N}^{p}\left(m_{1}\right), f_{N}^{p}\left(m_{3}\right)\right.\right.$, 
$\left.\left.f_{N}^{p}\left(m_{2}\right), \varepsilon\right)\right\}$ and $\exp \left\{t N L_{p}\left(f_{N}^{p}\left(m_{2}\right), f_{N}^{p}\left(m_{3}\right), f_{N}^{p}\left(m_{1}\right), \varepsilon\right)\right\}$ multiplied by some constant. Thus, taking into account (2.9), we obtain

$$
\begin{aligned}
& \sup _{m_{1}^{p}+m_{2}^{p}+m_{3}^{p}>3 \varepsilon} \mathbb{E}[ \exp \left\{H_{N}(t, \sigma)+H_{N}\left(t, \sigma^{\prime}\right)+H_{N}\left(t, \sigma^{\prime \prime}\right)-3 N t / 2\right\} \\
&\left.\times \mathbb{1}_{\left.\left\{H_{N}(t, \sigma)<N t(1+\varepsilon), H_{N}\left(t, \sigma^{\prime}\right)<N t(1+\varepsilon), H_{N}\left(t, \sigma^{\prime \prime}\right)<N t(1+\varepsilon)\right\}\right]}\right] \\
& \times \mathbb{P}_{m_{1}, m_{2}, m_{3}} \\
& \leq \sup _{m_{1}^{p}+m_{2}^{p}+m_{3}^{p}>3 \varepsilon} C_{5} \sqrt{N} \exp \left\{t N \operatorname { m i n } \left[Q\left(m_{1}^{p}, m_{2}^{p}, m_{3}^{p}, \varepsilon\right),\right.\right. \\
& L_{p}\left(m_{1}, m_{2}, m_{3}, \varepsilon\right), \\
&\left.L_{p}\left(m_{1}^{p}, m_{3}^{p}, m_{2}^{p}, \varepsilon\right), L_{p}\left(m_{2}^{p}, m_{3}^{p}, m_{1}^{p}, \varepsilon\right)\right] \\
&\left.\quad-N I\left(m_{1}, m_{2}, m_{2}\right)\right\}
\end{aligned}
$$

for all $t \in\left[0, T_{0}\right]$, where $C_{5}>0$ is a constant. Now the relevance of assumption (2.49) becomes clear. Due to (2.49), the right-hand side of (2.51) tends to 0 exponentially fast, as it can be estimated by $C_{5} \sqrt{N} \exp \left\{-h_{2} N\right\}$. The other terms in $\widetilde{I}_{N}^{4}(t, \varepsilon)$ have polynomial growth. Thus $\widetilde{I}_{N}^{4}(t, \varepsilon) \downarrow 0$ uniformly in $\left[T_{0}, T\right]$. This concludes the proof of the proposition.

PROPOSITION 2.6. For all $T>0$ satisfying (2.20) and all $\varepsilon>0$,

$$
\lim _{N \uparrow+\infty} N^{p-2} \mathbb{E}\left|\bar{V}_{N}(t, \varepsilon) \bar{Z}_{N}^{-2}(t)\right|=0
$$

uniformly in any interval $\left[T_{0}, T\right]$, where $0<T_{0}<T$.

Proof. It follows from the definition of $\bar{V}_{N}(t)$ that

$$
N^{p-2} \mathbb{E}\left|\bar{V}_{N}(t, \varepsilon) \bar{Z}_{N}^{-2}(t)\right| \leq \bar{C} N \mathbb{E} \frac{\mathbb{E}_{\sigma} e^{H_{N}(t, \sigma)} \mathbb{1}_{\left\{H_{N}(t, \sigma)>N t(1+\varepsilon)\right\}}}{\mathbb{E}_{\sigma} e^{H_{N}(t, \sigma)}}
$$

for all $t \geq 0$, where $\bar{C}>0$ is a constant. We will show that the expectation of this last fraction tends to 0 exponentially fast. First of all, we observe that, by (B.1),

$$
\begin{aligned}
& \frac{\mathbb{E E}_{\sigma} e^{H_{N}(t, \sigma)} \mathbb{1}_{\left\{H_{N}(t, \sigma)>N t(1+\varepsilon)\right\}}}{\mathbb{E E}_{\sigma} e^{H_{N}(t, \sigma)}} \\
& \quad=\mathbb{E} \mathbb{E}_{\sigma} e^{H_{N}(t, \sigma)-N t / 2} \mathbb{1}_{\left\{H_{N}(t, \sigma)>N t(1+\varepsilon)\right\}} \leq e^{-N t \varepsilon^{2} / 2} .
\end{aligned}
$$

Let us represent the fraction on the right-hand side of (2.53) as

$$
\begin{aligned}
\mathbb{E} & \frac{\mathbb{E}_{\sigma} e^{H_{N}(t, \sigma)} \mathbb{1}_{\left\{H_{N}(t, \sigma)>N t(1+\varepsilon)\right\}}}{\mathbb{E}_{\sigma} e^{H_{N}(t, \sigma)}} \\
= & \mathbb{E} \frac{\mathbb{E}_{\sigma} e^{H_{N}(t, \sigma)-N t / 2} \mathbb{1}_{\left\{H_{N}(t, \sigma)>N t(1+\varepsilon)\right\}}}{\exp \left\{\ln \mathbb{E}_{\sigma} e^{H_{N}(t, \sigma)}-\mathbb{E} \ln \mathbb{E}_{\sigma} e^{H_{N}(t, \sigma)}+\mathbb{E} \ln \mathbb{E}_{\sigma} e^{H_{N}(t, \sigma)}-N t / 2\right\}} .
\end{aligned}
$$


To expand this formula, we will use the concentration of measure as in (B.3). The random variable $\mathbb{E}_{\sigma} e^{H_{N}(t, \sigma)}$ has the same distribution as $\phi\left(J_{1}, \ldots, J_{\left(\begin{array}{l}N \\ p\end{array}\right)}\right)$, where the function

$$
\phi\left(x_{1}, \ldots, x_{\left(\begin{array}{c}
N \\
p
\end{array}\right)}\right)=\ln \mathbb{E}_{\sigma} \exp \left\{\sqrt{t N}\left(\begin{array}{c}
N \\
p
\end{array}\right)^{-1 / 2} \sum_{i_{1}<\cdots<i_{p}} x_{i_{1}, i_{2}, \ldots, i_{p}} \sigma_{i_{1}} \sigma_{i_{2}} \cdots \sigma_{i_{p}}\right\}
$$

is defined on $\mathbb{R}^{\left(\begin{array}{c}N \\ p\end{array}\right)}, J_{1}, \ldots, J_{\left(\begin{array}{c}N \\ p\end{array}\right)}$ are standard Gaussian random variables. The Lipschitz constant of $\phi\left(x_{1}, \ldots, x_{\left(\begin{array}{l}N \\ p\end{array}\right)}\right)$ is at most $\sqrt{t N}\left(\begin{array}{l}N \\ p\end{array}\right)^{-1 / 2}\left(\begin{array}{l}N \\ p\end{array}\right)^{1 / 2}=\sqrt{t N}$. Substituting this function and $u=N t \varepsilon^{2} / 4$ into (B.3), we derive

$$
\mathbb{P}\left\{\left|\ln \mathbb{E}_{\sigma} e^{H_{N}(t, \sigma)}-\mathbb{E} \ln \mathbb{E}_{\sigma} e^{H_{N}(t, \sigma)}\right|>N t \varepsilon^{2} / 4\right\} \leq e^{-N t \varepsilon^{4} / 32} .
$$

Let us introduce the events $O_{t, \varepsilon}^{N}:=\left\{\left|\ln \mathbb{E}_{\sigma} e^{H_{N}(t, \sigma)}-\mathbb{E} \ln \mathbb{E}_{\sigma} e^{H_{N}(t, \sigma)}\right|>N t \varepsilon^{2} / 4\right\}$. Consequently, by (2.55) and (2.56),

$$
\begin{aligned}
\mathbb{E} \frac{\mathbb{E}_{\sigma} e^{H_{N}(t, \sigma)} \mathbb{1}_{\left\{H_{N}(t, \sigma)>N t(1+\varepsilon)\right\}}}{\mathbb{E}_{\sigma} e^{H_{N}(t, \sigma)}} & \\
= & \mathbb{E} \frac{\mathbb{1}_{\left\{O_{t, \varepsilon}^{N}\right\}} \mathbb{E}_{\sigma} e^{H_{N}(t, \sigma)-N t / 2} \mathbb{1}_{\left\{H_{N}(t, \sigma)>N t(1+\varepsilon)\right\}}}{\exp \left\{\ln \mathbb{E}_{\sigma} e^{H_{N}(t, \sigma)}-\mathbb{E} \ln \mathbb{E}_{\sigma} e^{H_{N}(t, \sigma)}+\mathbb{E} \ln \mathbb{E}_{\sigma} e^{H_{N}(t, \sigma)}-N t / 2\right\}} \\
& +\mathbb{P}\left\{O_{t, \varepsilon}^{N}\right\} \\
\leq & e^{N t \varepsilon^{2} / 4} \mathbb{E} \frac{\mathbb{E}_{\sigma} e^{H_{N}(t, \sigma)-N t / 2} \mathbb{1}_{\left\{H_{N}(t, \sigma)>N t(1+\varepsilon)\right\}}}{\exp \left\{\mathbb{E} \ln \mathbb{E}_{\sigma} e^{H_{N}(t, \sigma)}-N t / 2\right\}}+e^{-N t \varepsilon^{4} / 32} .
\end{aligned}
$$

Observe that, for any $T$ satisfying (2.20) and any $0<T_{0}<T$, there exists a constant $K>0$ such that

$$
-K \sqrt{N}<\mathbb{E} \ln \mathbb{E}_{\sigma} e^{H_{N}(t, \sigma)}-N t / 2 \leq 0
$$

for all $t \in\left[T_{0}, T\right]$. The upper bound in (2.58) is immediate by the Jensen inequality. Whenever the second moment of $\bar{Z}_{N}(t)$ truncated is finite, the left-hand side of (2.58) was established by Talagrand [27] in the analysis of the critical temperature. We will outline his proof in our situation. For given $T$ satisfying (2.20), let us fix $\widetilde{\varepsilon}>0$ such that (2.48) and (2.49) hold. Let us define

$$
\bar{Z}_{N}(t, \widetilde{\varepsilon})=\mathbb{E}_{\sigma} e^{H_{N}(t, \sigma)-N t / 2} \mathbb{1}_{\left\{H_{N}(t, \sigma)<N t(1+\widetilde{\varepsilon})\right\}} .
$$

By (B.2) there exists a constant $K_{1}<0$ such that

$$
\mathbb{E} \bar{Z}_{N}(t, \widetilde{\varepsilon}) \geq K_{1}
$$

for all $t \in\left[T_{0}, T\right]$. Moreover, there exists a constant $K_{2}>0$ such that

$$
\mathbb{E} \bar{Z}_{N}^{3}(t, \widetilde{\varepsilon}) \leq K_{2}
$$


for all $t \in\left[T_{0}, T\right]$. The proof of (2.60) is analogous to the proof of the uniform convergence to 0 of $\widetilde{W}_{N}(t, \varepsilon)$ in Proposition 2.5. We decompose $\bar{Z}_{N}(t, \widetilde{\varepsilon})$ into four terms in the same way as we decomposed $\widetilde{W}_{N}(t, \varepsilon)$. The last three of them go to 0 uniformly in $t \in\left[T_{0}, T\right]$ and exponentially fast by the same arguments as $\widetilde{I}_{N}^{2}(t)$, and $\widetilde{I}_{N}^{3}(t)$ and $\widetilde{I}_{N}^{4}(t)$ do. We work out the first term similarly to the sum $I_{1}^{N}(t)+I_{2}^{N}(t)$ in Proposition 2.6. The only difference is the absence of terms in brackets in front of the exponent. The change $s_{i}=m_{i} \sqrt{N}$ makes the analogue of $I_{1}^{N}(t)$ tend to the integral over $\mathbb{R}^{3}$ of the density of three independent standard Gaussians, which equals 1 . Thus, in fact, $\bar{Z}_{N}(t, \widetilde{\mathcal{E}})$ converges to 1 uniformly in $\left[T_{0}, T\right]$ and (2.60) is obvious. Hence, for all $t \in\left[T_{0}, T\right]$,

$$
\frac{\mathbb{E} \bar{Z}_{N}^{2}(t, \widetilde{\varepsilon})}{\left(\mathbb{E} \bar{Z}_{N}(t, \widetilde{\varepsilon})\right)^{2}} \leq \frac{\left(\mathbb{E} \bar{Z}_{N}^{3}(t, \widetilde{\varepsilon})\right)^{2 / 3}}{\left(\mathbb{E} \bar{Z}_{N}(t, \widetilde{\varepsilon})\right)^{2}} \leq \frac{K_{2}^{2 / 3}}{K_{1}^{2}}:=K_{3} .
$$

Then starting from the Paley-Zygmund inequality and finally applying the concentration of measure inequality (B.3) with $u=N t / 2-\mathbb{E} \ln \mathbb{E}_{\sigma} e^{H_{N}(t, \sigma)}+$ $\ln \left(K_{1} / 2\right)$, we deduce

$$
\begin{aligned}
\frac{1}{4 K_{3}} \leq & \frac{\left(\mathbb{E} \bar{Z}_{N}(t, \widetilde{\varepsilon})\right)^{2}}{4 \mathbb{E} \bar{Z}_{N}^{2}(t, \widetilde{\varepsilon})} \\
\leq & \mathbb{P}\left\{\bar{Z}_{N}(t, \widetilde{\varepsilon})>\mathbb{E} \bar{Z}_{N}(t, \widetilde{\varepsilon}) / 2\right\} \\
\leq & \mathbb{P}\left\{\mathbb{E}_{\sigma} e^{H_{N}(t, \sigma)}>K_{1} e^{N t / 2} / 2\right\} \\
= & \mathbb{P}\left\{\ln \mathbb{E}_{\sigma} e^{H_{N}(t, \sigma)}-\mathbb{E} \ln \mathbb{E}_{\sigma} e^{H_{N}(t, \sigma)}\right. \\
& \left.>N t / 2-\mathbb{E} \ln \mathbb{E}_{\sigma} e^{H_{N}(t, \sigma)}+\ln \left(K_{1} / 2\right)\right\} \\
\leq & \exp \left\{\left[N t / 2-\mathbb{E} \ln \mathbb{E}_{\sigma} e^{H_{N}(t, \sigma)}+\ln \left(K_{1} / 2\right)\right]^{2} / 2 N t\right\},
\end{aligned}
$$

from which (2.58) follows. Finally, (2.54), (2.57) and (2.58) together imply

$$
\begin{aligned}
& \mathbb{E} \frac{\mathbb{E}_{\sigma} e^{H_{N}(t, \sigma)} \mathbb{1}_{\left\{H_{N}(t, \sigma)>N t(1+\varepsilon)\right\}}}{\mathbb{E}_{\sigma} e^{H_{N}(t, \sigma)}} \\
& \quad \leq e^{N t \varepsilon^{2} / 4+K \sqrt{N}} \mathbb{E}_{\sigma} e^{H_{N}(t, \sigma)-N t / 2} \mathbb{1}_{\left\{H_{N}(t, \sigma)>N t(1+\varepsilon)\right\}}+e^{-N t \varepsilon^{4} / 32} \\
& \quad \leq e^{-N t \varepsilon^{2} / 4+K \sqrt{N}}+e^{-N t \varepsilon^{4} / 32},
\end{aligned}
$$

and the proposition is proved.

ProOF OF (1.20). To complete the proof of Theorem 1.3, it remains to show that

$$
\lim _{p \uparrow+\infty} \inf _{m_{1}, m_{2}, m_{3} \in \mathcal{A}} Y_{p}\left(m_{1}, m_{2}, m_{3}\right)=2 \ln 2 .
$$


Making use of the functions $S\left(m_{1}, m_{2}, m_{3}\right)$ and $R\left(m_{1}, m_{2}, m_{3}\right)$, we get

$$
\begin{aligned}
& U_{p}\left(m_{1}, m_{2}, m_{3}\right) \\
& =I\left(m_{1}, m_{2}, m_{3}\right)\left(1+m_{3}^{p}\right) \\
& \quad \times\left[\left(4\left(1+m_{3}^{p}+\frac{m_{1}^{p}+m_{2}^{p}}{2}\right)^{2}+\frac{\left(m_{1}^{p}-m_{2}^{p}\right)^{2}\left(1+m_{3}^{p}\right)}{\left(1-m_{3}^{p}\right)}\right)^{1 / 2}\right. \\
& \left.\quad-\frac{\left(m_{1}^{p}-m_{2}^{p}\right)^{2}}{2\left(1-m_{3}^{p}\right)}-m_{1}^{p} m_{2}^{p}-\left(2+m_{3}^{p}\right)\right]^{-1} .
\end{aligned}
$$

It follows from (2.64) that, for any $p=2 k>2$ and any sequence $\left(m_{1, n}, m_{2, n}\right.$, $\left.m_{3, n}\right) \in \mathcal{A}$ such that $m_{1, n} \rightarrow 1, m_{2, n} \rightarrow 1, m_{3, n} \rightarrow 1$, as $n \rightarrow \infty, \lim _{n \uparrow+\infty}$ $\times Y_{p}\left(m_{1, n}, m_{2, n}, m_{3, n}\right)=2 \ln 2$. [In fact, by the definition of $\mathcal{A}$, we have ||$m_{1} \mid-$ $\left|m_{2}\right||\leq 1-| m_{3} \mid$ for all $\left(m_{1}, m_{2}, m_{3}\right) \in \mathcal{A}$, whence $\left(m_{1, n}^{p}-m_{2, n}^{p}\right)^{2}=o\left(1-m_{3, n}^{p}\right)$.] Thus $\lim \sup _{p \uparrow+\infty} \inf _{m_{1}, m_{2}, m_{3} \in A} Y_{p}\left(m_{1}, m_{2}, m_{3}\right) \leq 2 \ln 2$. This fact and the next proposition together imply (2.63).

Proposition 2.7. Let $\left\{p_{n}\right\}$ be a sequence of positive even numbers, $p_{n} \uparrow$ $+\infty$. Assume that the sequence $\left(m_{1, n}, m_{2, n}, m_{3, n}\right) \in \mathcal{A}$ satisfies one of the following conditions:

(i) $\left|m_{1, n}\right| \rightarrow 1,\left|m_{2, n}\right| \rightarrow 1,\left|m_{3, n}\right| \rightarrow 1$;

(ii) there exist $\delta>0$ and a pair $i$ and $j, i, j=1,2,3, i \neq j$, such that $\left|m_{i, n}\right| \rightarrow 1$ and $\left|m_{j, n}\right| \leq 1-\delta$ for all sufficiently large $n$;

(iii) there exists $\delta>0$ such that $\left|m_{1, n}\right| \leq 1-\delta,\left|m_{2, n}\right| \leq 1-\delta,\left|m_{3, n}\right| \leq 1-\delta$ for all sufficiently large $n$.

Then,

$$
\lim \inf _{n \uparrow+\infty} Y_{p_{n}}\left(m_{1, n}, m_{2, n}, m_{3, n}\right) \geq 2 \ln 2 .
$$

PROOF. In cases (i) and (iii) it suffices to substitute the sequence $\left(m_{1, n}, m_{2, n}\right.$, $\left.m_{3, n}\right)$ into the function $I\left(m_{1}, m_{2}, m_{3}\right)\left(2 / 3+\left(m_{1}^{p}+m_{2}^{p}+m_{3}^{p}\right)^{-1}\right)$. In case (ii) assume that, for example, $\left|m_{3, n}\right| \rightarrow 1$ and $\left|m_{1, n}\right| \leq 1-\delta$. Then $m_{1, n}^{p_{n}}=o(1)$. By definition of the set $\mathcal{A}$ we obtain ||$m_{1, n}|-| m_{2, n}|| \leq 1-\left|m_{3, n}\right| \rightarrow 0$ as $n \uparrow+\infty$; thus $m_{2, n}^{p_{n}}=o(1)$ and $\left(m_{1, n}^{p_{n}}-m_{2, n}^{p_{n}}\right)^{2} /\left(1-m_{3, n}^{p_{n}}\right)=o(1)$. Moreover, if $m_{3, n} \rightarrow 1$, then $m_{1, n}-m_{2, n} \rightarrow 0$ and if $m_{3, n} \rightarrow-1$, then $m_{1, n}+m_{2, n} \rightarrow 0$ and therefore in both of these cases $\liminf _{n \uparrow+\infty} I\left(m_{1, n}, m_{2, n}, m_{3, n}\right) \geq \ln 2$. This yields

$$
\begin{aligned}
\lim \inf _{n \uparrow+\infty} Y_{p_{n}}\left(m_{1, n}, m_{2, n}, m_{3, n}\right) & \geq \lim \inf _{n \uparrow+\infty} U_{p_{n}}\left(m_{1, n}, m_{2, n}, m_{3, n}\right) \\
& \geq \lim \inf _{n \uparrow+\infty} \ln 2 \frac{1+m_{3, n}^{p_{n}}}{m_{3, n}^{p_{n}}+o(1)} \geq 2 \ln 2,
\end{aligned}
$$

and the proposition is proved. 
PROOF OF THEOREM 1.4. From the discussion in the Introduction it remains to show $N^{(p-2) / 4} \ln \left(Z_{\beta, N}^{1} / \mathbb{E} Z_{\beta, N}\right) \rightarrow 0$ in probability as $N \uparrow \infty$, where $Z_{\beta, N}^{1}=$ $\mathbb{E}_{\sigma} e^{\beta \sqrt{N} X_{\sigma}^{1}}$ and

$$
X_{\sigma}^{1}=N^{-p / 2} \sum_{\begin{array}{c}
i_{1}, i_{2}, \ldots, i_{p} \\
\text { not all in pairs }
\end{array}} J_{i_{1}, i_{2}, \ldots, i_{p}} \sigma_{i_{1}} \sigma_{i_{2}} \cdots \sigma_{i_{p}} .
$$

Since $\mathbb{E} Z_{\beta, N} / \mathbb{E} Z_{\beta, N}^{1}=1+O\left(N^{(2-p) / 2}\right)$, one can concentrate on the convergence of the free energy $N^{(p-2) / 4} \ln \left(Z_{\beta, N}^{1} / \mathbb{E} Z_{\beta, N}^{1}\right)$. To prove the result up to Talagrand's bound, let us again truncate the Hamiltonian. Then

$$
\begin{aligned}
N^{(p-2) / 4} \ln \frac{Z_{\beta, N}^{1}}{\mathbb{E} Z_{\beta, N}^{1}}= & N^{(p-2) / 4} \ln \left(1+\frac{\widetilde{Z}_{\beta, N}^{1}-\mathbb{E} \widetilde{Z}_{\beta, N}^{1}}{\mathbb{E} Z_{\beta, N}^{1}}\right) \\
& +N^{(p-2) / 4} \ln \left(1+\frac{\widehat{Z}_{\beta, N}^{1}-\mathbb{E} \widehat{Z}_{\beta, N}^{1}}{Z_{\beta, N}-\left(\widehat{Z}_{\beta, N}^{1}-\mathbb{E} \widehat{Z}_{\beta, N}^{1}\right)}\right),
\end{aligned}
$$

where $\quad \widetilde{Z}_{\beta, N}^{1}=\mathbb{E}_{\sigma} e^{\beta \sqrt{N} X_{\sigma}^{1}} \mathbb{1}_{\left\{X_{\sigma}^{1}<\beta \sqrt{N}(1+\varepsilon)\right\}} \quad$ and $\quad \widehat{Z}_{\beta, N}^{1}=\mathbb{E}_{\sigma} e^{\beta \sqrt{N} X_{\sigma}^{1}} \times$ $\mathbb{1}_{\left\{X_{\sigma}^{1}>\beta \sqrt{N}(1+\varepsilon)\right\}}$. To treat the first term in (2.67), it suffices to show the convergence to 0 of

$$
\begin{aligned}
N^{(p-2) / 2} \operatorname{Var}\left(\frac{\widetilde{Z}_{\beta, N}^{1}-\mathbb{E} \widetilde{Z}_{\beta, N}^{1}}{\mathbb{E} Z_{\beta, N}^{1}}\right) \\
=\mathbb{E} \sum_{\substack{\sigma \cdot \sigma^{\prime}=m N, m=0,1 / N, \ldots, 1}}\left(e^{\beta \sqrt{N} X_{\sigma}^{1}+\beta \sqrt{N} X_{\sigma^{\prime}}^{1}-\beta^{2} N \mathbb{E}\left(X_{\sigma}^{1}\right)^{2}}\right. \\
\left.\quad \times \mathbb{1}_{\left\{X_{\sigma}^{1}<\beta \sqrt{N}(1+\varepsilon), X_{\sigma^{\prime}}^{1}<\beta \sqrt{N}(1+\varepsilon)\right\}}-1\right) \mathbb{P}_{m},
\end{aligned}
$$

where $\mathbb{P}_{m}=\mathbb{P}\left(\sigma \cdot \sigma^{\prime}=m N\right)$ with the asymptotics (2.24). It is not difficult to reduce this to the convergence of

$$
\begin{gathered}
N^{(p-2) / 2} \mathbb{E} \sum_{\substack{\sigma \cdot \sigma^{\prime}=m N,|m|<N^{-1 / 3-\delta}}}\left(e^{\beta \sqrt{N} X_{\sigma}^{1}+\beta \sqrt{N} X_{\sigma^{\prime}}^{1}-\beta^{2} N \mathbb{E}\left(X_{\sigma}^{1}\right)^{2}}-1\right) \mathbb{P}_{m} \\
=N^{(p-2) / 2} \sum_{\substack{\sigma \cdot \sigma^{\prime}=m N,|m|<N^{-1 / 3-\delta}}}\left(e^{\beta^{2} N f_{N}^{p}(m)}-1\right) \mathbb{P}_{m},
\end{gathered}
$$

with an arbitrary $0<\delta<1 / 6$ along the lines of Proposition 2.5. [Here $f_{N}^{p}\left(N^{-1}(\sigma\right.$. $\left.\left.\sigma^{\prime}\right)\right)=\mathbb{E}_{\sigma, \sigma^{\prime}} \operatorname{cov}\left(X_{\sigma}, X_{\sigma^{\prime}}\right)=\left(N^{-1}\left(\sigma \cdot \sigma^{\prime}\right)^{p}-N^{-p / 2}(p-1) ! !\right.$.] For this purpose, first for given $\beta$ smaller than Talagrand's bound (1.26) we choose $\varepsilon>0$ such 
that the power of the exponent in (2.28) is negative for all $m$ with $|m|>\varepsilon$. Thus the truncation makes the sum over $|m|>\varepsilon$ exponentially small. The next step is similar to that one for $\widetilde{I}_{N}^{2}(t)$ and $\widetilde{I}_{N}^{3}(t)$ in Proposition 2.5 by the use of the asymptotics $f_{N}^{p}(m)=o\left(m^{2}\right)$ and $\phi(m) \sim m^{2} / 2$ as $m \downarrow 0$. It reduces the problem to the analysis of the sum of untruncated Boltzmann weights with $|m|<\varepsilon^{2} / 4$, as it shows that the rest tends to 0 exponentially fast. The part of this last sum with $N^{-1 / 3-\delta}<|m|<\varepsilon^{2} / 4$ goes to 0 exponentially fast as well by the same arguments used for $I_{N}^{2}(t)$ in Proposition 2.3. Finally, to study (2.68), we replace $e^{\beta^{2} N f_{N}^{p}(m)}-1$ by its Taylor expansion. Then the sum over the first term is exponentially small. This is a consequence of the property of $X_{\sigma}^{1}$ :

$$
\begin{aligned}
& \sum_{m=0,1 / N, \ldots, N} f_{N}^{p}(m) \mathbb{P}_{m}=\mathbb{E}_{\sigma, \sigma^{\prime}} \operatorname{cov}\left(X_{\sigma}^{1}, X_{\sigma^{\prime}}^{1}\right) \\
& =N^{-p / 2} \sum_{\substack{i_{1}, \ldots, i_{p} \\
\text { not all in pairs }}} \mathbb{E}_{\sigma, \sigma^{\prime}} \sigma_{i_{1}} \sigma_{i_{1}}^{\prime} \cdots \sigma_{i_{p}} \sigma_{i_{p}}^{\prime}=0
\end{aligned}
$$

and of the fact that $\mathbb{P}_{m}$ is exponentially small for $|m|>N^{-1 / 3-\delta}$ with $\delta>1 / 6$. The sum over the second term of the Taylor expansion is of order at most $O\left(N^{2-2 p / 3-2 p \delta}\right)$ and multiplied by $N^{(p-2) / 2}$ converges to 0 for all $p \geq 3$ if $\delta>1 / 12$ is fixed.

To consider the second term in (2.67), one proves that $\mathbb{E}\left(\widehat{Z}_{\beta, N}^{1} / Z_{\beta, N}^{1}\right)$ and $\mathbb{E}\left(\mathbb{E} \widehat{Z}_{\beta, N}^{1} / Z_{\beta, N}^{1}\right)$ are exponentially small for any $\varepsilon>0$. This is completely analogous to (2.53) in Proposition 2.6; therefore we omit the details.

3. The fluctuations of the partition function in the REM. Amazingly enough, the simplest of all our models, the REM, will be seen to offer in some sense the most interesting behavior with regard to the fluctuations of the free energy. The main surprise here will be the existence of an intermediate region of temperatures where a CLT does not hold, but there a nonstandard limit theorem will be proven.

We begin with the proof of (i) of Theorem 1.5.

Proposition 3.1. Whenever $0<\beta<\sqrt{\ln 2 / 2}$,

$$
\exp \left\{\frac{N}{2}\left(\ln 2-\beta^{2}\right)\right\} \ln \frac{Z_{\beta, N}}{\mathbb{E} Z_{\beta, N}} \stackrel{\mathcal{D}}{\rightarrow} \mathcal{N}(0,1) .
$$

PROOF. This result will follow from the standard CLT for triangular arrays. Let us first write

$$
\ln \frac{Z_{\beta, N}}{\mathbb{E} Z_{\beta, N}}=\ln \left(1+\frac{Z_{\beta, N}-\mathbb{E} Z_{\beta, N}}{\mathbb{E} Z_{\beta, N}}\right) .
$$


We will show that the second term in the logarithm properly normalized will converge to a normal random variable. To see this, write

$$
\frac{Z_{\beta, N}-\mathbb{E} Z_{\beta, N}}{\mathbb{E} Z_{\beta, N}}=\sum_{\sigma \in \mathcal{S}_{N}} e^{-N\left(\ln 2+\beta^{2} / 2\right)}\left(e^{\beta \sqrt{N} X_{\sigma}}-e^{N \beta^{2} / 2}\right) \equiv \sum_{\sigma \in \mathcal{S}_{N}} y_{N}(\sigma) .
$$

Note that $\mathbb{E} y_{N}(\sigma)=0$ and $\mathbb{E} y_{N}^{2}(\sigma)=e^{-N\left(2 \ln 2-\beta^{2}\right)}\left[1-e^{-N \beta^{2}}\right]$ and thus

$$
\mathbb{E}\left(\frac{Z_{\beta, N}-\mathbb{E} Z_{\beta, N}}{\mathbb{E} Z_{\beta, N}}\right)^{2}=e^{-N\left(\ln 2-\beta^{2}\right)}\left[1-e^{-N \beta^{2}}\right] .
$$

Therefore we can write

$$
\frac{Z_{\beta, N}-\mathbb{E} Z_{\beta, N}}{\mathbb{E} Z_{\beta, N}}=\exp \left\{-\frac{N}{2}\left(\ln 2-\beta^{2}\right)\right\} \sqrt{1-e^{-N \beta^{2}}} \frac{1}{2^{N / 2}} \sum_{\sigma \in \delta_{N}} \widetilde{y}_{N}(\sigma),
$$

where $\widetilde{y}_{N}(\sigma)=\exp \left\{\frac{N}{2}\left(2 \ln 2-\beta^{2}\right)\right\}\left[1-e^{-N \beta^{2}}\right]^{-1 / 2} y_{N}(\sigma)$ has mean 0 and variance 1 . By the CLT for triangular arrays (see [25]), it follows readily that

$$
\frac{1}{2^{N / 2}} \sum_{\sigma \in \wp_{N}} \widetilde{y}_{N}(\sigma) \stackrel{D}{\rightarrow} \mathcal{N}(0,1)
$$

if the Lindeberg condition holds, that is, in this case, if, for any $\varepsilon>0$,

$$
\lim _{N \uparrow+0} \mathbb{E} \widetilde{y}_{N}^{2}(\sigma) \mathbb{1}_{\left\{\left|\widetilde{y}_{N}(\sigma)\right| \geq \varepsilon 2^{N / 2}\right\}}=0 .
$$

But

$$
\begin{aligned}
\mathbb{E} \widetilde{y}_{N}^{2}(\sigma) \mathbb{1}_{\left\{\left|\tilde{y}_{N}(\sigma)\right| \geq \varepsilon 2^{N / 2}\right\}} & \frac{1}{\sqrt{2 \pi}\left(1-e^{\left.-N \beta^{2}\right)}\right.} \\
& \times e^{-2 N \beta^{2}} \int_{\sqrt{N}(\ln 2 /(2 \beta)+\beta)+\ln \varepsilon /(\sqrt{N} \beta)+o(1 / \sqrt{N})}^{\infty} e^{2 \sqrt{N} \beta z-z^{2} / 2} d z+o(1) \\
= & \frac{1}{\sqrt{2 \pi}\left(1-e^{-N \beta^{2}}\right)} \int_{\sqrt{N}(\ln 2 /(2 \beta)-\beta)+\ln \varepsilon /(\sqrt{N} \beta)+o(1 / \sqrt{N})}^{\infty} e^{-z^{2} / 2} d z+o(1) .
\end{aligned}
$$

It is easy to check that the latter integral converges to 0 if and only if $\beta^{2}<\ln 2 / 2$. Using now the fact that $e^{x}=1+x+o(x)$ as $x \rightarrow 0$, it is now a trivial matter to deduce the assertion of the proposition.

Since the Lindeberg condition clearly fails for $2 \beta^{2} \geq \ln 2$, it is clear that we cannot expect a simple CLT beyond this regime. Such a failure of the CLT is always a problem related to "heavy tails" and results from the fact that extremal events begin to influence the fluctuations of the sum. It appears therefore reasonable to separate from the sum the terms where $X_{\sigma}$ is anomalously large. For Gaussian 
r.v.'s it is well known that the right scale of separation is given by $u_{N}(x)$ defined by

$$
2^{N} \int_{u_{N}(x)}^{\infty} \frac{d z}{\sqrt{2 \pi}} e^{-z^{2} / 2}=e^{-x}
$$

which (for $x>-\ln N / \ln 2$ ) is equal to (see, e.g., [17])

$$
u_{N}(x)=\sqrt{2 N \ln 2}+\frac{x}{\sqrt{2 N \ln 2}}-\frac{\ln (N \ln 2)+\ln 4 \pi}{2 \sqrt{2 N \ln 2}}+o\left(\frac{1}{\sqrt{N}}\right),
$$

where $x \in \mathbb{R}$ is a parameter. Let us now define

$$
Z_{N, \beta}^{x} \equiv \mathbb{E}_{\sigma} e^{\beta \sqrt{N} X_{\sigma}} \mathbb{1}_{\left\{X_{\sigma} \leq u_{N}(x)\right\}}
$$

We may write

$$
\begin{aligned}
\frac{Z_{\beta, N}-\mathbb{E} Z_{\beta, N}}{\mathbb{E} Z_{\beta, N}}= & 1+\frac{\mathbb{Z}_{\beta, N}^{x}-\mathbb{E} Z_{\beta, N}^{x}}{\mathbb{E} Z_{\beta, N}} \\
& +\frac{Z_{\beta, N}-Z_{\beta, N}^{x}-\mathbb{E}\left(Z_{\beta, N}-Z_{\beta, N}^{x}\right)}{\mathbb{E} Z_{\beta, N}} .
\end{aligned}
$$

Let us first consider the last summand. We introduce the random variable

$$
\mathcal{W}_{N}(x)=\frac{Z_{\beta, N}-Z_{\beta, N}^{x}}{\mathbb{E} Z_{\beta, N}}=e^{-N\left(\ln 2+\beta^{2} / 2\right)} \sum_{\sigma \in \delta_{N}} e^{\beta \sqrt{N} X_{\sigma}} \mathbb{1}_{\left\{X_{\sigma}>u_{N}(x)\right\}} .
$$

It will be convenient to rewrite this as [we ignore the subleading corrections to $u_{N}(x)$ and only keep the explicit representation (3.10)]

$$
\begin{aligned}
\mathcal{W}_{N}(x)= & \exp \left\{-N\left(\ln 2+\beta^{2} / 2\right)\right\} \sum_{\sigma \in \wp_{N}} \exp \left\{\beta \sqrt{N} u_{N}\left(u_{N}^{-1}\left(X_{\sigma}\right)\right)\right\} \mathbb{1}_{\left\{u_{N}^{-1}\left(X_{\sigma}\right)>x\right\}} \\
= & \exp \left\{-N\left(\ln 2+\beta^{2} / 2\right)\right\} \exp \left\{\beta N \sqrt{2 \ln 2}-\beta \frac{\ln (N \ln 2)+\ln 4 \pi}{2 \sqrt{2 \ln 2}}\right\} \\
& \times \sum_{\sigma \in S_{N}} \exp \left\{\frac{\beta}{\sqrt{2 \ln 2}} u_{N}^{-1}\left(X_{\sigma}\right)\right\} \mathbb{1}_{\left\{u_{N}^{-1}\left(X_{\sigma}\right)>x\right\}} .
\end{aligned}
$$

Let us now introduce the point process on $\mathbb{R}$ given by

$$
\mathcal{P}_{N} \equiv \sum_{\sigma \in \mathcal{S}_{N}} \delta_{u_{N}^{-1}\left(X_{\sigma}\right)}
$$

A classical result from the theory of extreme order statistics (see, e.g., [17]) asserts that the point process $\mathcal{P}_{N}$ converges weakly to a Poisson point process on $\mathbb{R}$ with intensity measure $e^{-x} d x$. We can, of course, write

$$
\sum_{\sigma \in S_{N}} \exp \left\{\frac{\beta}{\sqrt{2 \ln 2}} u_{N}^{-1}\left(X_{\sigma}\right)\right\} \mathbb{1}_{\left\{u_{N}^{-1}\left(X_{\sigma}\right)>x\right\}} \stackrel{\mathcal{D}}{\rightarrow} \int_{x}^{\infty} e^{\alpha z} \mathcal{P}_{N}(d z),
$$


where we set $\alpha \equiv \beta / \sqrt{2 \ln 2}$. Clearly, the weak convergence of $\mathcal{P}_{N}$ to $\mathcal{P}$ implies convergence in law of the right-hand side of (3.16), provided that $e^{\alpha x}$ is integrable on $[x, \infty)$ w.r.t. the Poisson process with intensity $e^{-x}$. This is, in fact, never a problem: the Poisson point process has almost surely support on a finite set, and therefore $e^{\alpha x}$ always a.s. integrable. Note, however, that for $\beta \geq \sqrt{2 \ln 2}$ the mean of the integral is infinite, indicating the passage to the low-temperature regime. Note also that the variance of the integral is finite exactly if $\alpha<1 / 2$, that is, $\beta^{2}<\ln 2 / 2$, that is, when the CLT holds. On the other hand, the mean of the integral diverges if $x \downarrow-\infty$; note that at $-\infty$ the points of the Poisson point process accumulate, and there is no finite support argument as before that would assure the existence if $x$ is taken to $-\infty$. The following lemma provides the first step in the proof of part (ii) of Theorem 1.5 and of Theorem 1.6.

Lemma 3.2. Let $\mathfrak{W}_{N}(x), \alpha$ be defined as above and let $\mathcal{P}$ be the Poisson point process with intensity measure $e^{-z} d z$. Then

$$
\exp \left\{\frac{N}{2}(\sqrt{2 \ln 2}-\beta)^{2}+\frac{\alpha}{2}[\ln (N \ln 2)+\ln 4 \pi]\right\} \mathcal{W}_{N}(x) \stackrel{D}{\rightarrow} \int_{x}^{\infty} e^{\alpha z} \mathcal{P}(d z) .
$$

REMARK. Note that the mean of the right-hand side is finite if and only if $\beta<\sqrt{2 \ln 2}$. Thus only in that case does this lemma also allow one to deal with the centered variable appearing in (3.12).

We now need to turn to the remaining term,

$$
\frac{Z_{\beta, N}^{x}-\mathbb{E} Z_{\beta, N}^{x}}{\mathbb{E} Z_{\beta, N}}=\frac{\mathcal{V}_{N}(x)}{\mathbb{E} Z_{\beta, N}},
$$

where

$$
\mathcal{V}_{N}(x) \equiv Z_{\beta, N}^{x}-\mathbb{E} Z_{\beta, N}^{x} .
$$

One might first hope that this term upon proper scaling would converge to a Gaussian; however, one can easily check that this is not the case (the Lindeberg condition will not be verified). However, it will not be hard to compute all moments of this term:

Lemma 3.3. Let $\mathcal{V}_{N}(x)$ be defined by (3.19). Then, for $\alpha>1 / 2$ and any integer $k \geq 2$,

$$
\begin{aligned}
& \lim _{N \uparrow+\infty} \frac{\mathbb{E}\left[\mathcal{V}_{N}(x)\right]^{k}}{\left[2^{-N} \exp \left\{N \beta \sqrt{2 \ln 2}-\frac{\alpha}{2}[\ln (N \ln 2)+\ln 4 \pi]\right\}\right]^{k}} \\
&=\sum_{i=1}^{k} \frac{1}{i !} \sum_{\substack{\ell_{1} \geq 2, \ldots, \ell_{i} \geq 2 \\
\sum_{j} \ell_{j}=k}} \frac{k !}{\ell_{1} ! \cdots \ell_{i} !} \frac{e^{(k \alpha-i) x}}{\left(\ell_{1} \alpha-1\right) \cdots\left(\ell_{i} \alpha-1\right)} .
\end{aligned}
$$


For $\alpha=1 / 2$, we have, for $k$ even,

$$
\lim _{N \uparrow+\infty} \frac{\mathbb{E}\left[\mathcal{V}_{N}(x)\right]^{k}}{\left[2^{-N} e^{N \beta \sqrt{2 \ln 2}}\right]^{k}}=\frac{k !}{(k / 2) ! 2^{k}}=\frac{(k-1) ! !}{2^{k / 2}}
$$

and, for $k$ odd,

$$
\lim _{N \uparrow+\infty} \frac{\mathbb{E}\left[\mathcal{V}_{N}(x)\right]^{k}}{\left[2^{-N} e^{N \beta \sqrt{2 \ln 2}}\right]^{k}}=0
$$

(which are the moments of the normal distribution with variance 1/2).

PROOF. This is a pure computation. Set $T_{N}(\sigma) \equiv e^{\beta \sqrt{N} X_{\sigma}} \mathbb{1}_{\left\{X_{\sigma} \leq u_{N}(x)\right\}}$. Note that, for $\beta<\sqrt{2 \ln 2}$,

$$
\begin{aligned}
\mathbb{E} T_{N}(\sigma) & =\int_{-\infty}^{u_{N}(x)} \frac{d z}{\sqrt{2 \pi}} \exp \left\{-\frac{z^{2}}{2}+\beta \sqrt{N} z\right\} \\
& =\exp \left\{\frac{N \beta^{2}}{2}\right\}\left(1-\int_{u_{N}(x)-\beta \sqrt{N}}^{\infty} \frac{d z}{\sqrt{2 \pi}} \exp \left\{-\frac{z^{2}}{2}\right\}\right) \sim \exp \left\{\frac{\beta^{2} N}{2}\right\},
\end{aligned}
$$

while, for $\beta>\sqrt{2 \ln 2}$ and all $k \geq 1$ and for $\beta>\sqrt{\ln 2 / 2}$ and for $k \geq 2$,

$$
\begin{aligned}
\mathbb{E}\left[T_{N}(\sigma)\right]^{k} & =\int_{-\infty}^{u_{N}(x)} \frac{d z}{\sqrt{2} \pi} \exp \left\{-\frac{z^{2}}{2}+k \beta \sqrt{N} z\right\} \\
& =\exp \left\{\frac{N k^{2} \beta^{2}}{2}\right\} \int_{-\infty}^{u_{N}(x)-k \beta \sqrt{N}} \frac{d z}{\sqrt{2} \pi} \exp \left\{-\frac{z^{2}}{2}\right\} \\
& \sim \exp \left\{\frac{N k^{2} \beta^{2}}{2}\right\} \frac{e^{-\left(u_{N}(x)-k \beta \sqrt{N}\right)^{2} / 2}}{\sqrt{2 \pi}\left(k \beta \sqrt{N}-u_{N}(x)\right)} \\
& \sim \frac{2^{-N} e^{-x}}{k \alpha-1} \exp \left\{k\left[\beta \sqrt{2 \ln 2} N+\alpha x-\frac{\alpha}{2}[\ln (N \ln 2)+\ln 4 \pi]\right]\right\} .
\end{aligned}
$$

Formula (3.24) is also valid for $\beta=\sqrt{2 \ln 2}$ with $k>1$ and for $\beta=\sqrt{\ln 2 / 2}$ with $k>2$. It is easy to see from the computations above that, for $\beta=\sqrt{2 \ln 2}$ with $k=1$ and also for $\beta=\sqrt{\ln 2 / 2}$ with $k=2$, we have

$$
\mathbb{E}\left[T_{N}(\sigma)\right]^{k} \sim \frac{e^{k^{2} \beta^{2} N / 2}}{2}=\frac{2^{-N} e^{-x}}{2} e^{k[\beta \sqrt{2 \ln 2} N+\alpha x]} .
$$

We set $\widetilde{T}_{N}(\sigma) \equiv 2^{-N} T_{N}(\sigma)$; by (3.24) we get, for $\beta>\sqrt{\ln 2 / 2}$ with $k \geq 2$ and also for $\beta>\sqrt{2 \ln 2}$ with $k \geq 1$,

$$
\begin{array}{r}
\mathbb{E}\left[\widetilde{T}_{N}(\sigma)\right]^{k} \sim \frac{2^{-N} e^{-x}}{k \alpha-1} \exp \{k[\beta \sqrt{2 \ln 2} N-N \ln 2+\alpha x \\
\left.\left.-\frac{\alpha}{2}[\ln (N \ln 2)+\ln 4 \pi]\right]\right\} .
\end{array}
$$


This formula is also true for $\beta=\sqrt{\ln 2 / 2}, k>2$ and $\beta=\sqrt{2 \ln 2}, k>1$. For $\beta=\sqrt{2 \ln 2}$ and $k=1$ and also for $\beta=\sqrt{\ln 2 / 2}$ and $k=2$ by (3.25),

$$
\mathbb{E}\left[\widetilde{T}_{N}(\sigma)\right]^{k} \sim \frac{2^{-N} e^{-x}}{2} e^{k[\beta \sqrt{2 \ln 2} N-N \ln 2+\alpha x]} .
$$

Now

$$
\begin{aligned}
\mathbb{E}\left[\mathcal{V}_{N}(x)\right]^{k}= & \mathbb{E}\left(\sum_{\sigma \in \delta_{N}}\left[\widetilde{T}_{N}(\sigma)-\mathbb{E} \widetilde{T}_{N}(\sigma)\right]\right)^{k} \\
= & \sum_{\sigma_{1}, \ldots, \sigma_{k} \in \delta_{N}} \mathbb{E} \prod_{i=1}^{k}\left[\widetilde{T}_{N}\left(\sigma_{i}\right)-\mathbb{E} \widetilde{T}_{N}\left(\sigma_{i}\right)\right] \\
= & \sum_{i=1}^{k} \sum_{\substack{\ell_{1}, \ldots, \ell_{i} \geq 2 \\
\sum_{j} \ell_{j}=k}} \frac{k !}{\ell_{1} ! \cdots \ell_{i} !}\left(\begin{array}{c}
2^{N} \\
i
\end{array}\right) \\
& \quad \times \mathbb{E}\left[\widetilde{T}_{N}(\sigma)-\mathbb{E} \widetilde{T}_{N}(\sigma)\right]^{\ell_{1}} \cdots \mathbb{E}\left[\widetilde{T}_{N}(\sigma)-\mathbb{E} \widetilde{T}_{N}(\sigma)\right]^{\ell_{i}} .
\end{aligned}
$$

Note finally that, for $l \geq 2$ and $\beta \geq \sqrt{\ln 2 / 2}$,

$$
\begin{aligned}
& \mathbb{E}\left[\widetilde{T}_{N}(\sigma)-\mathbb{E} \widetilde{T}_{N}(\sigma)\right]^{\ell} \\
& \quad=\sum_{j=1}^{\ell}(-1)^{j}\left(\begin{array}{l}
\ell \\
j
\end{array}\right) \mathbb{E} \widetilde{T}_{N}(\sigma)^{\ell-j}\left[\mathbb{E} \widetilde{T}_{N}(\sigma)\right]^{j} \sim \mathbb{E} \widetilde{T}_{N}(\sigma)^{\ell} .
\end{aligned}
$$

In fact, if $\sqrt{\ln 2 / 2} \leq \beta<\sqrt{2 \ln 2}, l \geq 2, j \geq 1, j \neq l-1, l$, then, by (3.23) and (3.26) and (3.27),

$$
\frac{\mathbb{E}\left[T_{N}^{l-j}(\sigma)\right]\left[\mathbb{E} T_{N}(\sigma)\right]^{j}}{\mathbb{E}\left[T_{N}^{l}(\sigma)\right]}=e^{N j\left(\beta^{2} / 2-\beta \sqrt{2 \ln 2}\right)} O\left(N^{\alpha j / 2}\right) .
$$

For $l \geq 2, j=l-1, l$,

$$
\begin{aligned}
\frac{\mathbb{E}\left[T_{N}^{l-j}(\sigma)\right]\left[\mathbb{E} T_{N}(\sigma)\right]^{j}}{\mathbb{E}\left[T_{N}^{l}(\sigma)\right]} & =e^{N l\left(\beta^{2} / 2-\beta \sqrt{2 \ln 2}\right)+N \ln 2} O\left(N^{\alpha l / 2}\right) \\
& \leq e^{-N \ln 2 / 2} O\left(N^{\alpha l / 2}\right) .
\end{aligned}
$$

For $\beta \geq \sqrt{2 \ln 2}, l \geq 2$ and $j \geq 1$ by (3.26) and (3.27),

$$
\frac{\mathbb{E}\left[T_{N}^{l-j}(\sigma)\right]\left[\mathbb{E} T_{N}(\sigma)\right]^{j}}{\mathbb{E}\left[T_{N}^{l}(\sigma)\right]}=O\left(2^{-N}\right) .
$$


Thus, for $l \geq 2$ and $\beta>\sqrt{\ln 2 / 2}$ and also for $l \geq 3$ and $\beta=\sqrt{\ln 2 / 2}$,

$$
\begin{aligned}
\mathbb{E}\left[\widetilde{T}_{N}(\sigma)-\mathbb{E} \widetilde{T}_{N}(\sigma)\right]^{\ell} & \\
\sim \frac{2^{-N} e^{-x}}{k \alpha-1} & {\left[2^{-N} \exp \{N \beta \sqrt{2 \ln 2}\} \exp \{\alpha x\}\right.} \\
& \left.\times \exp \left\{-\frac{\alpha}{2}[\ln (N \ln 2)+\ln 4 \pi]\right\}\right]^{\ell} .
\end{aligned}
$$

Inserting this result into (3.28) gives the assertion of the lemma, namely, (3.20).

For $\beta=\sqrt{\ln 2 / 2}$ and $l=2$ by (3.27), we have

$$
\mathbb{E}\left[\widetilde{T}_{N}(\sigma)-\mathbb{E} \widetilde{T}_{N}(\sigma)\right]^{2} \sim \frac{2^{-N} e^{-x}}{2}\left[2^{-N} e^{N \beta \sqrt{2 \ln 2}} e^{\alpha x}\right]^{2} .
$$

Inserting this formula into (3.28), we see that the term with $l_{1}, \ldots, l_{i}=2, i=k / 2$ brings the main contribution to the sum, and all others are of smaller order, because of the polynomial terms $\exp \{-l \alpha / 2 \ln (N \ln 2)\}$ in (3.33). This implies (3.21) and (3.22) and the lemma is proved.

REMARK. One sees that if we let $x \downarrow-\infty$, and rescale properly, the corresponding moments converge to that of a centered Gaussian r.v. This could alternatively be seen by checking that the Lindeberg condition holds for the truncated variables provided $x \leq-2 \ln \ln 2^{N}$.

A standard consequence of Lemma 3.3 is the weak convergence of the normalized version of $\mathcal{V}_{N}(x)$ :

COROLlary 3.4. For $\sqrt{\ln 2 / 2}<\beta$,

$$
\exp \left\{\frac{N}{2}(\sqrt{2 \ln 2}-\beta)^{2}+\frac{\alpha}{2}[\ln (N \ln 2)+\ln 4 \pi]\right\} \frac{\mathcal{V}_{N}(x)}{\mathbb{E} Z_{\beta, N}} \stackrel{D}{\rightarrow} \mathcal{V}(x, \alpha),
$$

where $\mathcal{V}(x, \alpha)$ is the random variable with mean 0 and $k$-th moments given by the right-hand side of (3.20). For $\beta=\sqrt{\ln 2 / 2}$,

$$
\sqrt{2} \exp \left\{\frac{N}{2}(\sqrt{2 \ln 2}-\beta)^{2}\right\} \frac{\mathcal{V}_{N}(x)}{\mathbb{E} Z_{\beta, N}} \stackrel{D}{\rightarrow} \mathcal{N}(0,1) .
$$

The next proposition will imply (ii) of Theorem 1.5.

Proposition 3.5. Let $\sqrt{\ln 2 / 2}<\beta<\sqrt{2 \ln 2}$. Then, for $x \in \mathbb{R}$ chosen arbitrarily,

$$
\begin{aligned}
\exp \{ & \left.\frac{N}{2}(\sqrt{2 \ln 2}-\beta)^{2}+\frac{\alpha}{2}[\ln (N \ln 2)+\ln 4 \pi]\right\} \ln \frac{Z_{\beta, N}}{\mathbb{E} Z_{\beta, N}} \\
& \stackrel{D}{\rightarrow} \mathcal{V}(x, \alpha)+\int_{x}^{\infty} e^{\alpha z} \mathcal{P}(d z)-\int_{x}^{\infty} e^{\alpha z} e^{-z} d z,
\end{aligned}
$$

where $\mathcal{V}(x, \alpha)$ and $\mathcal{P}$ are independent random variables. 
PROOF. Equation (3.37) would be immediate from Lemma 3.2 and Corollary 3.4 if $\mathcal{W}_{N}(x)$ and $\mathcal{V}_{N}(x)$ were independent. However, while this is not true, they are not far from independent. To see this, note that if we condition on the number of variables $X_{\sigma}, n_{N}(x)$, that exceed $u_{N}(x)$, the decomposition in (3.12) is independent. On the other hand, one readily verifies that Corollary 3.4 also holds under the conditional law $\mathbb{P}\left[\cdot \mid n_{N}(x)=n\right]$, for any finite $n$, with the same right-hand side $\mathcal{V}(x, \alpha)$. But this implies that the limit can be written as the sum of two independent random variables, as desired.

Since, for $\beta^{2}>\ln 2 / 2, \alpha>1 / 2, \mathbb{E} \mathcal{V}(x, \alpha)^{2}=e^{x(2 \alpha-1)} /(2 \alpha-1)$ tends to 0 as $x \downarrow-\infty$, therefore we see that

$$
\mathcal{V}(x, \alpha)=\mathbb{D} \lim _{y \uparrow+\infty} \int_{-y}^{x} e^{\alpha z} \mathcal{P}(d z)-\int_{-y}^{x} e^{\alpha z} e^{-z} d z,
$$

which means that we can make sense out of the Poisson integral $\int_{-\infty}^{\infty} e^{\alpha z}(\mathcal{P}(d z)-$ $e^{-z} d z$ ). We see that Propositions 3.1 and 3.5 imply Theorem 1.5.

REMARK. The appearance of the intermediate region with non-Gaussian fluctuations may appear surprising in view of the fact that in the $p$-spin models we could prove the CLT up to a much higher value of $\beta$, in fact up to almost the critical value. The reason, however, lies in the fact that in the $p$-spin model the Gaussian part of the fluctuation is always on a polynomial scale in $N$, while the truncation error $\left(\left(Z_{\beta, N}-Z_{\beta, N}^{T}\right) / \mathbb{E} Z_{\beta, N}\right)$ is exponentially small even when we truncate at $\beta(1+\varepsilon) \sqrt{N}$, way below where we truncate in the REM. This means that the CLT contribution will always dominate the extremal fluctuations. In the REM everything is exponentially small, and while a sufficiently truncated partition function gives a Gaussian contribution, this is dominated by the larger extremal fluctuations in the intermediate regime. In other words, the extra correlations in the $p$-spin models strengthen the Gaussian fluctuations more than the extremal ones, which sounds intuitive.

PROOF OF THEOREM 1.6. We will see that the computations above almost suffice to conclude the low-temperature case as well. With the notation from above, we write

$$
Z_{\beta, N}=Z_{\beta, N}^{x}+\left(Z_{\beta, N}-Z_{\beta, N}^{x}\right) .
$$

Clearly, for $\beta \geq \sqrt{2 \ln 2}$,

$$
\begin{aligned}
Z_{\beta, N}-Z_{\beta, N}^{x}= & \exp \left\{N[\beta \sqrt{2 \ln 2}-\ln 2]-\frac{\alpha}{2}[\ln (N \ln 2)+\ln 4 \pi]\right\} \\
& \times \sum_{\sigma \in S_{N}} \mathbb{1}_{\left\{u_{N}^{-1}(\sigma)>x\right\}} \exp \left\{\alpha u_{N}^{-1}\left(X_{\sigma}\right)\right\}
\end{aligned}
$$


so that, for any $x \in \mathbb{R}$,

$$
\begin{aligned}
& \left(Z_{\beta, N}-Z_{\beta, N}^{x}\right) \exp \left\{-N[\beta \sqrt{2 \ln 2}-\ln 2]+\frac{\alpha}{2}[\ln (N \ln 2)+\ln 4 \pi]\right\} \\
& \stackrel{\mathcal{D}}{\rightarrow} \int_{x}^{\infty} e^{\alpha z} \mathcal{P}(d z) .
\end{aligned}
$$

Now write

$$
Z_{\beta, N}^{x}=\mathbb{E} Z_{\beta, N}^{x}\left(1+\frac{Z_{\beta, N}^{x}-\mathbb{E} Z_{\beta, N}^{x}}{\mathbb{E} Z_{\beta, N}^{x}}\right) .
$$

Let us first treat the case $\beta>\sqrt{2 \ln 2}$. By (3.24) we have

$$
\mathbb{E} Z_{\beta, N}^{x} \sim \frac{2^{-N} e^{-x}}{\alpha-1} \exp \left\{\beta \sqrt{2 \ln 2} N+\alpha x-\frac{\alpha}{2}[\ln (N \ln 2)+\ln 4 \pi]\right\} .
$$

Thus

$$
\begin{gathered}
\exp \left\{-N[\beta \sqrt{2 \ln 2}-\ln 2]+\frac{\alpha}{2}[\ln (N \ln 2)+\ln 4 \pi]\right\} Z_{\beta, N}^{x} \\
=\frac{e^{x(\alpha-1)}}{\alpha-1}\left(1+\frac{Z_{\beta, N}^{x}-\mathbb{E} Z_{\beta, N}^{x}}{\mathbb{E} Z_{\beta, N}^{x}}\right)(1+o(1)) .
\end{gathered}
$$

Using Lemma 3.3, we see that now

$$
\frac{Z_{\beta, N}^{x}-\mathbb{E} Z_{\beta, N}^{x}}{\mathbb{E} Z_{\beta, N}^{x}} \frac{e^{x(\alpha-1)}}{\alpha-1}
$$

converges in distribution to a random variable with moments given by the right-hand side of (3.20). Moreover, as $x \downarrow-\infty$, this variable converges to 0 in probability. Since the same is true for the prefactor, the assertion of the theorem is now immediate.

Let us consider now the case $\beta=\sqrt{2 \ln 2}$. Proceeding as in (3.24),

$$
\begin{aligned}
\mathbb{E} Z_{\beta, N}^{0} & =\frac{2^{N}}{\sqrt{2 \pi}} \int_{-\infty}^{u_{N}(0)-\sqrt{2 N \ln 2}} e^{-z^{2} / 2} d z \\
& =2^{N}\left(\frac{1}{2}-\frac{\ln (N \ln 2)+\ln 4 \pi}{4 \sqrt{N \pi \ln 2}}+O\left(\frac{(\ln N)^{2}}{N}\right)\right) .
\end{aligned}
$$

We use the decomposition

$$
Z_{\beta, N}=Z_{\beta, N}-Z_{\beta, N}^{0}+\mathbb{E} Z_{\beta, N}^{0}+\left(Z_{\beta, N}^{0}-\mathbb{E} Z_{\beta, N}^{0}\right) .
$$

By (3.45), $\mathbb{E} Z_{\beta, N}^{0} / \mathbb{E} Z_{\beta, N} \sim 1 / 2$. By (3.14), we easily see that

$$
\frac{Z_{\beta, N}-Z_{\beta, N}^{0}}{\mathbb{E} Z_{\beta, N}}=\mathcal{W}_{N}(x) \rightarrow 0 \text { a.s. }
$$


even though $\mathbb{E} \mathfrak{W}_{N}(0)=1 / 2$ ! Thus the more precise statement consists of saying that

$$
e^{[\ln (N \ln 2)+\ln 4 \pi] / 2} \mathcal{W}_{N}(0) \stackrel{\mathcal{D}}{\rightarrow} \int_{0}^{\infty} e^{z} \mathcal{P}(d z) .
$$

Note that, of course, the limiting variable has infinite mean, but is a.s. finite. Finally, by Corollary 3.4,

$$
e^{[\ln (N \ln 2)+\ln 4 \pi] / 2} \frac{Z_{\beta, N}^{0}-\mathbb{E} Z_{\beta, N}^{0}}{\mathbb{E} Z_{\beta, N}} \stackrel{D}{\rightarrow} \mathcal{V}(0,1) .
$$

The same arguments as those given after Proposition 3.5 allow us to identify $\mathcal{V}(0,1)$ with the centered Poisson integral $\int_{-\infty}^{0} e^{z}\left(\mathcal{P}(d z)-e^{-z} d z\right)$. This implies (1.31). Equation (1.32) is an immediate corollary. This concludes the proof of Theorem 1.6.

\section{APPENDIX A}

More general covariance functions. Let $X$ be a Gaussian vector on the hypercube $S_{N}=\{-1,1\}^{N}$ with mean 0 and covariances $\operatorname{cov}\left(X_{\sigma}, X_{\sigma^{\prime}}\right)=$ $f_{N}^{p}\left(R_{N}\left(\sigma, \sigma^{\prime}\right)\right) \equiv \sum_{k=0}^{[p / 2]} \alpha_{p-2 k}(N) R_{N}\left(\sigma, \sigma^{\prime}\right)^{p-2 k}$, where $\alpha_{p-2 k}(N) \sim \alpha_{p-2 k} N^{-k}$ as $N \uparrow \infty$. Assume that $n$ is the minimal positive integer number such that $\mathbb{E}_{\sigma, \sigma^{\prime}}\left(\operatorname{cov}\left(X_{\sigma}, X_{\sigma^{\prime}}\right)\right)^{n} \neq 0$. Then $\mathbb{E}_{\sigma, \sigma^{\prime}}\left(\operatorname{cov}\left(X_{\sigma}, X_{\sigma^{\prime}}\right)\right)^{n} \sim C N^{-n p / 2}$.

CONJECTURE. Let $p>2$ and define $Z_{N, \beta}=\mathbb{E}_{\sigma} e^{\beta \sqrt{N} X_{\sigma}}$. There exists $\beta_{c}>0$ such that, for all $\beta<\beta_{c}$,

$$
N^{n(p-2) / 4} \ln \frac{Z_{\beta, N}}{\mathbb{E} Z_{\beta, N}} \stackrel{\mathcal{D}}{\rightarrow} \mathcal{N}\left(0, \beta^{2 n} C / n !\right)
$$

Moreover, the constant $C$ can be computed as follows. Here $n$ is also the minimal positive integer such that $\mathbb{E}\left(\sum_{k=0}^{[p / 2]} \alpha_{p-2 k} \xi^{p-2 k}\right)^{n} \neq 0$, where $\xi$ is a standard Gaussian random variable and $C=\mathbb{E}\left(\sum_{k=0}^{[p / 2]} \alpha_{p-2 k} \xi^{p-2 k}\right)^{n}$.

Trying to prove this conjecture by the martingale method of [9] in the same way as in Theorem 1.2, one successfully arrives at the analogue of (2.21) where the scaling factor is $N^{n(p-2) / 2}$ and $V_{N}(t)=\mathbb{E}_{\sigma, \sigma^{\prime}}\left(N f_{N}^{p}\left(R_{N}\left(\sigma, \sigma^{\prime}\right)\right)-\right.$ $\left.t^{n-1} N^{n(2-p) / 2} C /(n-1) !\right) e^{H_{N}(t, \sigma)+H_{N}\left(t, \sigma^{\prime}\right)}$. By the same arguments as for (2.23), one shows that $N^{n(p-2) / 2} \mathbb{E} V_{N}(t) \rightarrow 0$ (just substitute the Taylor expansion of $e^{t N f_{N}^{p}(m)}$ up to the $n$th order).

However, to get rid of the absolute value in (2.21), we apply the CauchySchwarz inequality and pass to the third moment of the partition function. Then, because of the nonindependence of three random variables $\sigma \cdot \sigma^{\prime}, \sigma \cdot \sigma^{\prime \prime}$ and $\sigma^{\prime} \cdot \sigma^{\prime \prime}$, 
the terms (2.44) and (2.45) will not be small in a general situation. Consider the example of a process such as (1.4) where the summation is over the sets of $p$ indices $\left\{i_{1}, \ldots, i_{p}\right\}$ with at most two indices equal. Then $n=2$ but the term (2.44) is a constant. Similarly, in the model (1.4) of Theorem 1.4 (the summation over all possible sets of indices) with $p$ odd, we have $n=2$, the term (2.45) equals 0 , all other terms obtained from the second-order expansion are small and only the term (2.44) is large. To overcome this difficulty, one could use the CauchySchwarz inequality in a different way passing to the fourth moment of the partition function. But then very tough computations will be required.

\section{APPENDIX B}

Two useful theorems. We state two useful results for the convenience of the reader. The first concerns standard estimates for truncated exponential moments of Gaussian random variables.

FACT B.1. Let $\xi$ be a Gaussian random variable with $\mathbb{E} \xi=0, \mathbb{E} \xi^{2}=1$. Then, for all $a, b>0$,

$$
\begin{aligned}
& \mathbb{E}\left[\exp \{a \xi\} \mathbb{1}_{\{\xi>b\}}\right] \leq \frac{1}{\sqrt{2 \pi(b-a)}} \exp \left\{-b^{2} / 2+a b\right\} \quad \text { if } b>a \\
& \mathbb{E}\left[\exp \{a \xi\} \mathbb{1}_{\{\xi<b\}}\right] \leq \frac{1}{\sqrt{2 \pi(a-b)}} \exp \left\{-b^{2} / 2+a b\right\} \quad \text { if } b<a
\end{aligned}
$$

The second is the Gaussian concentration of measure inequality, to be found, for example, in [18].

FACT B.2. Assume that $f\left(x_{1}, \ldots, x_{d}\right)$ is a function on $\mathbb{R}^{d}$ with a Lipschitz constant $L$. Let $J_{1}, \ldots, J_{d}$ be independent standard Gaussian random variables. Then, for any $u>0$,

$$
\mathbb{P}\left\{\left|f\left(J_{1}, \ldots, J_{d}\right)-\mathbb{E} f\left(J_{1}, \ldots, J_{d}\right)\right|>u\right\} \leq \exp \left\{-u^{2} /\left(2 L^{2}\right)\right\} .
$$

Acknowledgments. The authors thank Francis Comets for valuable comments and, in particular, for pointing out the fact that in the $\tilde{X}_{\sigma}$ model the CLT is due to the spin-independent term in (1.24). This work was supported in part by the Deutsche Forschungsgemeinschaft in the program "Interacting Stochastic Systems of high Complexity."

\section{REFERENCES}

[1] Aizenman, M., Lebowitz, J. L. and Ruelle, D. (1987). Some rigorous results on Sherrington-Kirkpatrick spin glass model. Comm. Math. Phys. 112 3-20. 
[2] Aizenman, M. and Wehr, J. (1990). Rounding effects of quenched randomness of first-order phase transitions. Comm. Math. Phys. 130 489-528.

[3] Bovier, A. (1998). The Kac version of the Sherrington-Kirkpatrick model at high temperatures. J. Statist. Phys. 91 459-474.

[4] Bovier, A. (2001). Statistical Mechanics of Disordered Systems. MaPhySto Lecture Notes 10. Univ. Aarhus.

[5] Bovier, A. and GAyrard, V. (1996). The retrieval phase of the Hopfield model: a rigorous analysis of the overlap distribution. Probab. Theory Related Fields 107 61-98.

[6] Bovier, A., Gayrard, V. and Picco, P. (1995). Gibbs states of the Hopfield model with extensively many patterns. J. Statist. Phys. 79 395-414.

[7] Bovier, A. and MASOn, D. (2001). Extreme value behavior in the Hopfield model. Ann. Appl. Probab. 11 91-120.

[8] Comets, F. (1996). A spherical bound for the Sherrington-Kirkpatrick model. Hommage à P. A. Meyer et J. Neveu. Astérisque 236 103-108.

[9] Comets, F. and Neveu, J. (1995). The Sherrington-Kirkpatrick model of spin glasses and stochastic calculus: the high temperature case. Comm. Math. Phys. 166 549-564.

[10] DerridA, B. (1980). Random energy model: limit of a family of disordered models. Phys. Rev. Lett. 45 79-82.

[11] DERRIDA, B. (1981). Random energy model: an exactly solvable model of disordered systems. Phys. Rev. B 24 2613-2626.

[12] Dorlas, T. C. and Wedagedera, J. R. (2001). Large deviations and the random energy model. Internat. J. Modern Phys. B 15 1-15.

[13] Eisele, Th. (1983). On a third order phase transition. Comm. Math. Phys. 90 125-159.

[14] Galvez, A., Martinez, S. and Picco, P. (1989). Fluctuations in Derrida's random energy and generalized random energy models. J. Statist. Phys. 54 515-529.

[15] Gardner, E. (1985). Spin glasses with p-spin interactions. Nuclear Phys. B 257 747-765.

[16] JACOD, J. and ShiryaEv, A. N. (1987). Limit Theorems for Stochastic Processes. Springer, Berlin.

[17] Leadbetter, M. R., Lindgren, G. and Rootzén, H. (1983). Extremes and Related Properties of Random Sequences and Processes. Springer, Berlin.

[18] Ledoux, M. and Talagrand, L. (1991). Probability in Banach Space. Springer, Berlin.

[19] Newman, CH. M. and Stein, D. L. (1997). Thermodynamic chaos and the structure of short range spin glasses. In Mathematical Aspects of Spin Glasses and Neural Networks (A. Bovier and P. Picco, eds.). Birkhäuser, Boston.

[20] De Oliveira, V. and Fontanari, J. F. (1999). Replica analysis of the $p$-spin interaction Ising spin-glass model. J. Phys. A 32 2285-2296.

[21] Olivieri, E. and PiCCO, P. (1991). On the existence of thermodynamics for the random energy model. Comm. Math. Phys. 96 125-144.

[22] Revuz, D. and Yor, M. (1992). Brownian Motion and Continuous Martingales. Springer, Berlin.

[23] Ruelle, D. (1987). A mathematical reformulation of Derrida's REM and GREM. Comm. Math. Phys. 108 225-239.

[24] Sherrington, D. and Kirkpatrick, S. (1972). Solvable model of a spin glass. Phys. Rev. Lett. 35 1792-1796.

[25] Shiryaev, A. N. (1996). Probability, 2nd ed. Springer, New York.

[26] Talagrand, M. (1995). Concentration of measure and isoperimetric inequalities in product space. Inst. Hautes Études Sci. Publ. Math. 81 73-205. 
[27] TAlagrand, M. (2000). Rigorous low temperature results for mean field $p$-spin interaction models. Probab. Theory Related Fields 117 303-360.

[28] Toubol, A. (1998). High temperature regime for a multidimensional Sherrington-Kirkpatrick model of spin glass. Probab. Theory Related Fields 110 497-534.

\section{A. BOVIER}

WEIERSTRASS-INSTITUT FÜR ANGEWANDTE

ANALYSIS UND STOCHASTIK

MOHRENSTRASSE 39

D-10117 BERLIN

GERMANY

E-MAIL: bovier@wias-berlin.de
I. KURKOVA

UFR DE MATHEMATIQUES

UNIVERSITÉ PARIS 6

4 PLACE JUSSIEU

F-75252 PARIS CEDEX 05

FRANCE

E-MAIL: kourkova@ccr.jussieu.fr

M. LÖWE

MATHEMATICS

KATHOLIEKE UNIVERSITEIT NIJMEGEN

Postbus 9010

6500 GL NIJMEGEN

THE NETHERLANDS

E-MAIL: loewe@sci.kun.nl 